Research Paper

\title{
Finite element modelling of fracture propagation in saturated media using quasi-zero-thickness interface elements
}

\author{
Ignasi de-Pouplana ${ }^{\mathrm{a}, *}$, Eugenio Oñate ${ }^{\mathrm{a}, \mathrm{b}}$ \\ ${ }^{a}$ CIMNE - Centre Internacional de Metodes Numerics en Enginyeria, Gran Capitán s/n, 08034 Barcelona, Spain \\ b Departament d'Enginyeria Civil i Ambiental (DECA), Universitat Politècnica de Catalunya (UPC), Campus Nord, C. Jordi Girona, 1-3, 08034 Barcelona, Spain
}

\section{A R T I C L E I N F O}

\section{Keywords:}

Coupled solid-pore fluid formulation

Non-local damage model

Interface elements

Finite element method

Finite increment calculus

\begin{abstract}
A B S T R A C T
A new computational technique for the simulation of $2 \mathrm{D}$ and $3 \mathrm{D}$ fracture propagation processes in saturated porous media is presented. A non-local damage model is conveniently used in conjunction with interface elements to predict the degradation pattern of the domain and insert new fractures followed by remeshing. FICstabilized elements of equal order interpolation in the displacement and the pore pressure have been successfully used under complex conditions near the undrained-incompressible limit. A bilinear cohesive fracture model describes the mechanical behaviour of the joints. A formulation derived from the cubic law models the fluid flow through the crack. Examples in 2D and 3D, using 3-noded triangles and 4-noded tetrahedra respectively, are presented to illustrate the accuracy and robustness of the proposed methodology.
\end{abstract}

\section{Introduction}

Modelling the fluid flow in a multi-fractured porous domain implies taking into account that the cracks in the solid skeleton introduce preferential flow paths as well as jumps in the displacement field. A proper understanding of discontinuities is crucial not only because they influence the behaviour of the local surroundings of the cracks, but also because they modify the global permeability and the mechanical response of the medium, specially whenever it undergoes a crack growth process.

Numerical methods that allow analysing and understanding the complexity of a multi-fractured porous domain are of major interest in various fields, but probably the most widely known is the petroleum industry. Here, the oil-gas-soil interaction takes the leading role along with the hydraulic fracturing as a common technique to enhance reservoir permeability and well efficiency [1,2]. Other possible applications can be found in the geothermal energy production, where the solid-pore fluid formulation is coupled with the thermal problem [3], the underground storage of carbon dioxide [4], and even the study of fractures in epithelial cell sheets $[5,6]$.

In the last decades, important efforts have been made to develop numerical models for the accurate analysis of discontinuities in solids and porous media.

The extended finite element method (XFEM) has obtained notable attention in the past years [7-10]. The discontinuity is captured by means of enrichment functions that introduce the jumps in the displacement field.
The most noticeable advantage of the method is that there is no need to explicitly represent cracks in a mesh, provided that enriched nodes are considered. This avoids the necessity of remeshing during crack growth, but in return it demands a higher computational cost in terms of number of degrees of freedom and numerical integration.

The present work focuses on the numerical techniques purely based on the finite element method (FEM). In this category, numerous methods can be found in the literature, but two main subgroups can be distinguished: the "smeared crack" and the "discrete crack" approaches. The former can be classed as continuum based methods in which the influence of developing fractures is incorporated into the constitutive stress-strain law [11-14]. In discrete crack models, however, each single discontinuity is represented explicitly [15-18]. In this paper, the heterogeneity of materials is considered through the combination of standard elements governed by a smeared crack model, with interface elements governed by a discrete crack model.

Since Goodman et al. proposed the "zero-thickness" interface element to describe the mechanical behaviour of pre-existing joints in rock masses [19] many authors have developed strategies to adapt this element for the solution of fracture processes in coupled solid-pore fluid problems.

Three different types of zero-thickness interface elements can be found in the literature concerning the way the fluid is modelled: single, double and triple noded elements. The single-noded element is the simplest one and only considers longitudinal conductivity with no pressure drop across the interface [20]. The triple-noded element was

\footnotetext{
* Corresponding author at: CIMNE, Edificio C1, Campus Nord, UPC, Gran Capitán s/n, 08034 Barcelona, Spain.

E-mail address: ipouplana@cimne.upc.edu (I. de-Pouplana).
} 


$\begin{array}{ll}\text { Nomenclature } \\ \text { Symbols } & \\ \alpha & \text { Biot's coefficient } \\ \delta & \text { relative displacements of the interface } \\ \lambda & \text { rotation matrix } \\ \sigma & \text { total stress vector } \\ \sigma^{\prime} & \text { effective stress vector } \\ \varepsilon & \text { strain vector } \\ \boldsymbol{k} & \text { intrinsic permeability matrix } \\ \boldsymbol{u} & \text { displacement vector } \\ \breve{\varepsilon}_{e q} & \text { non-local equivalent strain } \\ \delta_{c} & \text { critical relative displacement of the interface } \\ \epsilon & \text { volumetric strain of the solid skeleton } \\ \kappa & \text { compressive to tensile strength ratio } \\ \mathbb{d} & \text { damage } \\ \mathbb{d}_{p} & \text { fracture propagation damage } \\ \mathscr{D} & \text { distance } \\ \mu & \text { dynamic viscosity } \\ \mu_{F} & \text { friction coefficient of the interface } \\ \nu & \text { Poisson's ratio } \\ \phi & \text { porosity } \\ \rho & \text { density of the solid-fluid mixture } \\ \rho_{f} & \text { fluid density }\end{array}$

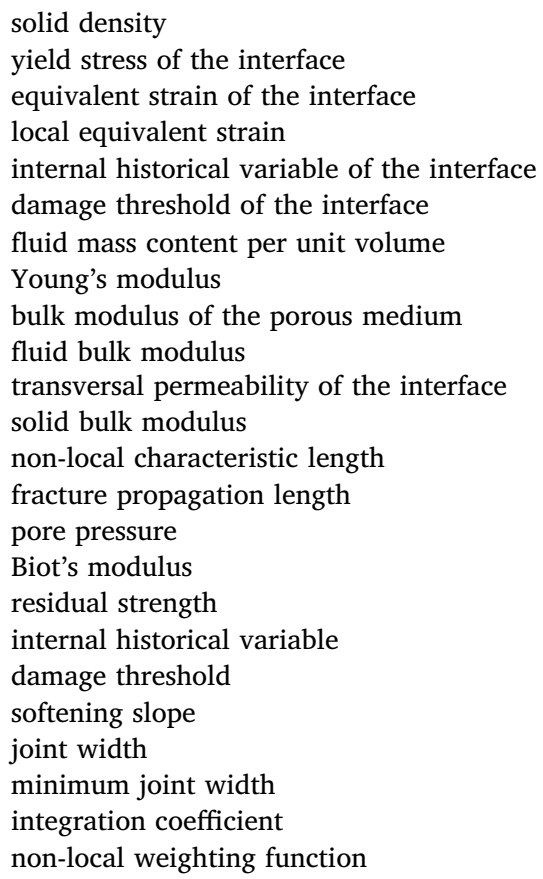

meant to include the effect of the transversal conductivity through the discontinuity [21]. The two nodes at each side of the interface represent the potentials in the pore pressure, while the third node, placed at the middle of the interface, stores the average potential of the longitudinal fluid through the fracture. Finally, the double-noded elements take into account both types of conductivity but the external nodes variables substitute the middle node. $\mathrm{Ng}$ and Small [22] used this double-noded zero-thickness interface element to model flow problems with pre-existing discontinuities, but did not consider hydraulic potential drop between the two interface walls. Segura and Carol [23] introduced the transversal conductivity in double-noded zero-thickness elements to account for the exchange of fluid between the discontinuity and the porous media.

Regarding the mechanical behaviour of fractures, there are basically two different approaches: those based on linear elastic fracture mechanics (LEFM), and those based on non-linear fracture mechanics (NLFM). LEFM techniques were first proposed to solve fracture propagation problems by means of remeshing without considering a fracture process zone (FPZ) before the crack tip. This approach is applicable in large structures where the size of the FPZ is negligible. However, for quasi-brittle analyses, the consideration of a non-linear fracture process zone where the energy is dissipated before it completely fails was found to be essential. In those cases the NLFM technique is usually applied and a softening law relates the cohesive stress to the crack opening in the FPZ. The first procedure based on the cohesive fracture model was originally introduced by Barenblatt $[24,25]$ for brittle materials and by Dugdale [26] for plastic materials. Hillerborg et al. [27] developed the first fictitious crack model for Mode I fracture. It was extended later for the mixed mode fracture, from which Camacho and Ortiz [28] proposed a suitable fracture criterion that is widely used in the literature.

One of the most important parts in the modelling of fracture propagation is the criterion for determining the direction of the crack growth. Some methodologies are based on the local evaluation of the stress field at the crack tip, such as the maximum circumferential stress [29] and the maximum principal stress criteria [30,31]. Others measure the energy distribution at the fractured zone, e.g. the minimum strain energy density criterion [32] or the maximum strain energy release rate criterion [33]. Finally, some authors have developed crack growth criteria based on continuum damage mechanics [34] and, more recently, combined with level set procedures $[35,36]$.

In order to reduce mesh-induced directional bias, in this work we use a non-local damage model in combination with a discrete crack approach in which discontinuities are represented by quasi-zerothickness interface elements. A special remeshing technique allows us introducing new joint elements according to the damage map obtained with the damage model. The low permeability and high compressibility of this kind of problems makes the pressure field oscillate spuriously if equal order interpolation elements are used without stabilization. Here we solve the solid-pore fluid interaction problem with a FIC-FEM stabilized formulation presented in a recent work by the authors [37].

The paper is organized as follows. First, we present the coupled FICFEM formulation derived from the second-order FIC form of the mass balance equation in space. Next, the developed methodology for fracture propagation is introduced, explaining the fundamental theory behind the non-local damage model, and describing the quasi-zerothickness interface elements. Finally, two academic plane-strain examples are solved to test the accuracy of the proposed methodology. Finally, one additional three-dimensional case is included to show the performance of the generalized 3D formulation.

\section{Solid-pore fluid FIC-FEM formulation}

In 1941 Biot [38] found a relation between the fluid mass content per unit volume $\zeta$, the volumetric strain of the solid skeleton $\epsilon$ and the pore pressure $p$ as

$\zeta=\alpha \in+\frac{p}{Q}$

In the above relation, $\alpha$ is the Biot's coefficient and $Q$ is a combined compressibility of the fluid-solid phases, also called Biot's modulus [39]:

$\alpha=1-\frac{K}{K_{s}} \leqslant 1 ; \quad \frac{1}{Q}=\frac{\alpha-\phi}{K_{s}}+\frac{\phi}{K_{f}}$

being $\phi$ the porosity of the soil, $K_{s}$ the bulk modulus of the solid phase, $K_{f}$ the bulk modulus of the fluid phase, and $K$ the bulk modulus of the 
porous medium, which can be computed from the Young's modulus $E$ and the Poisson's ratio $v$ as

$K=\frac{E}{3(1-2 v)}$

Terzaghi [40] also showed the importance of the effective stress $\sigma_{i j}^{\prime}$ as responsible for the major deformations and rupture of the solid skeleton. It can be defined as

$\sigma_{i j}^{\prime}=\sigma_{i j}+\alpha p \delta_{i j}$

where $\sigma_{i j}$ is the total stress.

The behaviour of a saturated porous medium can be described by combining two equations: the balance of momentum for the mixture solid-fluid and the mass balance for the pore fluid.

Balance of momentum for the mixture solid-fluid

$r_{u}:=\frac{\partial}{\partial x_{j}}\left(\sigma_{i j}^{\prime}-\alpha p \delta_{i j}\right)+\rho b_{i}-\rho \ddot{u}_{i}=0$

where $b_{i}$ is the body force per unit mass, $\ddot{u}_{i}$ is the acceleration of the points in the solid skeleton and $\rho$ is the density of the solid-fluid mixture $\rho=\phi \rho_{f}+(1-\phi) \rho_{s}$, being $\rho_{f}$ the density of the fluid and $\rho_{s}$ the density of the solid.

Mass balance for the pore fluid

$r_{p}:=\alpha \dot{\epsilon}+\frac{\dot{p}}{Q}+\frac{\partial}{\partial x_{i}}\left[-\frac{1}{\mu} k_{i j}\left(\frac{\partial p}{\partial x_{j}}-\rho_{f} b_{j}\right)\right]=0$

where $\mu$ is the dynamic viscosity of the fluid and $k_{i j}$ is the intrinsic permeability matrix of the porous medium.

The system of partial differential Eqs. (5) and (6) allows us obtaining the displacements $u_{i}$ and the pressure $p$ fields. However, under undrained-incompressible conditions, i.e. when $k_{i j} \rightarrow 0$ and $Q \rightarrow \infty$, the systems becomes ill-posed and the spaces used to approximate the displacement and pressure fields must fulfil the Babuska-Brezzi conditions to avoid locking of the pressure field [41,42].

The present work uses linear elements of equal order interpolation for the displacement and pressure fields, together with a stabilized form of the mass balance equation based on the Finite Increment Calculus (FIC method) [43-47]. Essentially, the original mass balance Eq. (6) is substituted by the second-order FIC form of the mass balance equation in space [48], given by

$\widehat{r}_{p}:=r_{p}+\frac{h_{i}^{2}}{12} \frac{\partial^{2} r_{p}}{\partial x_{i}^{2}}=0$

in which $h_{i}$ are space dimensions related to characteristic element dimensions. Note that for the standard infinitesimal form of the mass balance equation is recovered.

The balance of momentum Eq. (5) and the FIC form of the continuity Eq. (7) are discretized in space by interpolating the displacement and pressure fields as: $\boldsymbol{u}=\boldsymbol{N}_{u} \overline{\boldsymbol{u}}$ and $p=\boldsymbol{N}_{p} \overline{\boldsymbol{p}}$ where $\overline{(\cdot)}$ denotes nodal values and

$\boldsymbol{N}_{u}=\left[\begin{array}{ccccccc}N_{1} & 0 & 0 & \ldots & N_{n} & 0 & 0 \\ 0 & N_{1} & 0 & \ldots & 0 & N_{n} & 0 \\ 0 & 0 & N_{1} & \ldots & 0 & 0 & N_{n}\end{array}\right]$

$\boldsymbol{N}_{p}=\left[N_{1}, \ldots, N_{n}\right]$

We apply the standard Galerkin technique, using $\boldsymbol{N}_{u}^{T}$ and $\boldsymbol{N}_{p}^{T}$ as test functions, and integrate over the domain to obtain the following stabilized system of equations written in Voigt notation:

$\boldsymbol{r}_{u}=\boldsymbol{M} \ddot{\ddot{\boldsymbol{u}}}+\int_{\Omega} \boldsymbol{B}^{T} \boldsymbol{\sigma}^{\prime} d \Omega-\boldsymbol{Q \boldsymbol { p }}-\boldsymbol{f}_{u}=\mathbf{0}$
$\widehat{\boldsymbol{r}}_{p}=\left(\boldsymbol{Q}^{T}-\boldsymbol{R}\right) \dot{\overline{\boldsymbol{u}}}-\boldsymbol{l}+\left(\boldsymbol{C}+\boldsymbol{T}-\boldsymbol{T}_{b}\right) \dot{\overline{\boldsymbol{p}}}+\boldsymbol{H} \overline{\boldsymbol{p}}-\boldsymbol{f}_{p}=\mathbf{0}$

with the standard matrix and vector terms:

$\boldsymbol{M}=\int_{\Omega} \boldsymbol{N}_{u}^{T} \rho \boldsymbol{N}_{u} d \Omega ; \quad \boldsymbol{Q}=\int_{\Omega} \boldsymbol{B}^{T} \alpha \boldsymbol{m} \boldsymbol{N}_{p} d \Omega$

$\boldsymbol{C}=\int_{\Omega} \boldsymbol{N}_{p}^{T} \frac{1}{Q} \boldsymbol{N}_{p} d \Omega ; \quad \boldsymbol{H}=\int_{\Omega}\left(\nabla \boldsymbol{N}_{p}\right)^{T} \frac{1}{\mu} \boldsymbol{k} \nabla \boldsymbol{N}_{p} d \Omega$

$\boldsymbol{f}_{u}=\int_{\Omega} \boldsymbol{N}_{u}^{T} \rho \boldsymbol{b} d \Omega+\int_{\Gamma_{t}} \boldsymbol{N}_{u}^{T} \tilde{\boldsymbol{t}} d \Gamma$

$\boldsymbol{f}_{p}=\int_{\Omega}\left(\nabla \boldsymbol{N}_{p}\right)^{T} \frac{1}{\mu} \boldsymbol{k} \rho_{f} \boldsymbol{b} d \Omega-\int_{\Gamma_{q}} \boldsymbol{N}_{p}^{T} \widetilde{q}_{n} d \Gamma$

Here $\boldsymbol{B}$ is the matrix of shape functions' derivatives, $\widetilde{\boldsymbol{t}}$ is the vector of prescribed surface tractions, $\widetilde{q}_{n}$ is the prescribed normal flow rate, and the vector $\boldsymbol{m}$ comes from the definition of the effective stresses $\sigma^{\prime}=\sigma+\alpha p m$ with:

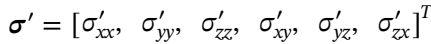

$\boldsymbol{m}=\left[\begin{array}{lllll}1, & 1, & 1, & 0,0,0\end{array}\right]^{T}$

The stabilizing terms in Eqs. (10) and (11) are:

$\boldsymbol{l}=\int_{\Omega}\left(\nabla \boldsymbol{N}_{p}\right)^{T} \frac{\tau}{3} \hat{\boldsymbol{S}}^{T} \dot{\sigma}^{\prime} d \Omega ; \quad \boldsymbol{R}=\int_{\Omega}\left(\nabla \boldsymbol{N}_{p}\right)^{T} \tau 2 G \boldsymbol{S}^{T} \boldsymbol{I}_{v} \boldsymbol{B} d \Omega$

$\boldsymbol{T}=\int_{\Omega}\left(\nabla \boldsymbol{N}_{p}\right)^{T} \tau\left(\alpha-\frac{2 G}{3 \alpha Q}\right)\left(\nabla \boldsymbol{N}_{p}\right) d \Omega$

$\boldsymbol{T}_{b}=\int_{\Gamma} \boldsymbol{N}_{p}^{T} \frac{\tau}{h_{n}} \frac{4 G}{3 \alpha Q} \boldsymbol{N}_{p} d \Gamma$

All the above matrices and vectors can be computed at elemental level and subsequently assembled in the global equations system, as in the standard FEM [49]. A detailed derivation of the stabilized equations and a complete explanation of the different terms can be found in [37].

\section{Modelling of discontinuities in porous media}

This section is organized in three parts. First, the main concepts behind the damage model used in the porous domain are introduced. Then, the formulation of the interface elements is presented. Finally, we explain how both approaches are combined in this work.

\subsection{Continuum damage model}

We have chosen the simple isotropic damage model with a single scalar variable. This model, which holds for multiaxial stress states, is based on the assumptions that the stiffness degradation is isotropic and the Poisson's ratio is not affected by damage. The stress-strain law in Voigt notation is postulated as:

$\sigma=(1-\mathbb{d}) \boldsymbol{D}_{e} \varepsilon=(1-\mathbb{d}) \sigma_{e}$

where $\boldsymbol{D}_{e}$ is the elastic constitutive matrix, $\sigma_{e}$ is the undamaged stress vector, and $d$ is the damage variable, a normalized scalar measure of the material degradation, ranging from 0 to 1 .

In order to properly determine the evolution of the damage variable regardless of the loading case, we introduce a historical variable $r(t)$ defined as:

$r(t)=\max \left\{r_{y}, \max _{\tau \leqslant t} \varepsilon_{e q}(\tau)\right\}$

In the above expression $\varepsilon_{e q}$ is the equivalent strain, i.e. a scalar measure of the strain level, and $r_{y}$ is the damage threshold, a material parameter that indicates the equivalent strain value at which damage starts.

\subsubsection{Equivalent strain}

To some extent, the equivalent strain presented in (22) plays a role similar to the yield function in plasticity as it directly affects the shape 
of the elastic domain. There are numerous forms for the equivalent strain in the literature. A convenient choice for quasi-brittle materials, such as rock or concrete, is the so called modified von Mises definition [50], i.e.

$\varepsilon_{e q}=\frac{\kappa-1}{2 \kappa(1-2 \nu)} I_{1}+\frac{1}{2 \kappa} \sqrt{\left(\frac{\kappa-1}{1-2 \nu} I_{1}\right)^{2}+\frac{12 \kappa}{(1+\nu)^{2}} J_{2}}$

where $\kappa$ is a model parameter that sets the ratio between the uniaxial compressive strength and the uniaxial tensile strength, $I_{1}$ is the first invariant of the strain tensor and $J_{2}$ is the second invariant of the deviatoric strain tensor.

\subsubsection{Damage evolution law}

There are various damage governing laws that can be effectively used to model damage growth in quasi-brittle materials. Here we use the exponential softening model proposed in [51]:

$g(r)=1-\frac{r_{y}(1-R)}{r}-R \exp \left\{-S\left(r-r_{y}\right)\right\}$

In the above expression, the parameter $R$ is associated to the residual strength of the material, whereas the parameter $S$ controls the slope of the softening branch after the peak of the stress-strain curve.

Essentially, the damage variable $\mathbb{d}$ is computed from the combination of Eqs. (22) and (24) as

$\mathbb{d}=g(r)$ with $\left\{\begin{array}{cc}g(r)=0 & \text { if } r=r_{y} \\ 0<g(r) \leq 1 & \text { if } r>r_{y}\end{array}\right.$

\subsubsection{Non-local damage model}

Problems involving damage progression in quasi-brittle materials show strong localization of strains. If the damage variable depends only on the strain state at the point under consideration and no regularization is introduced, numerical simulations exhibit a pathological mesh dependence and the energy consumed by the fracture process tends to zero as the mesh is refined [52]. The introduction of a characteristic length into the constitutive model, and the formulation of a non-local strain-softening model, have been shown to prevent the spurious localization of strains and regularize the boundary value problem [53-55]. An integral-type non-local damage model has been used in this work for that purpose.

In essence, such model abandons the classical assumption of locality and admits that the damage at a certain point depends not only on the state variables at that point, but also on the distribution of the state variables over a finite neighbourhood of the point under consideration.

Damage in this work has been computed from the so-called nonlocal equivalent strain [56]. Let $\varepsilon_{e q}(\boldsymbol{x})$ be the local equivalent strain in a domain $\Omega$. The corresponding non-local strain field is defined as:

$\varepsilon_{e q, n l}(\boldsymbol{x}) \equiv \breve{\varepsilon}_{e q}(\boldsymbol{x})=\int_{\Omega} Z(\boldsymbol{x}, \chi) \varepsilon_{e q}(\chi) d \chi$

where $Z(\boldsymbol{x}, \chi)$ is the non-local weighting function:

$Z(\boldsymbol{x}, \chi)=\frac{Z_{0}(\|\boldsymbol{x}-\chi\|)}{\int_{\Omega} Z_{0}(\|\boldsymbol{x}-\varphi\|) d \varphi}$

and $Z_{0}$ is defined as the following Gauss distribution function:

$Z_{0}(\|\boldsymbol{x}-\chi\|)=Z_{0}\left(\mathscr{D}_{x \chi}\right)= \begin{cases}\exp \left[-\left(\frac{2 \mathscr{T}_{x x}}{l_{c}}\right)^{2}\right] & \text { if } \mathscr{D}_{x \chi} \leqslant l_{c} \\ 0 & \text { if } \mathscr{D}_{x \chi}>l_{c}\end{cases}$

where $\mathscr{D}_{x \chi}$ is the distance between points $\boldsymbol{x}$ and $\chi$, and $l_{c}$ is the characteristic length, a material parameter reflecting the internal length of the non-local continuum. The function in (28) implies that any point $\chi$ within a radius $l_{c}$ of the source point $\boldsymbol{x}$ has an influence on the value of the non-local equivalent strain in $\boldsymbol{x}$.

The non-local equivalent strain in Eq. (26) substitutes the local

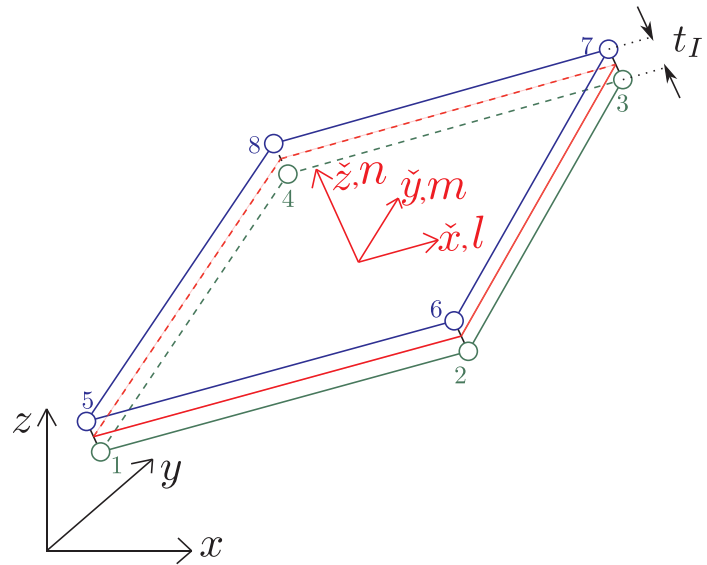

Fig. 1. Scheme of a generic hexahedral interface element. Global and local coordinate system.

equivalent strain in Eq. (22).

\subsection{Quasi-zero-thickness interface elements}

The quasi-zero-thickness interface elements developed in this work follow the same idea as the double-noded zero-thickness interface elements of [22] or [23] but, in fact, they are surface elements in 2D and volume elements in 3D. Hence, they can be defined using a width larger than zero if necessary.

One of the most important differences with respect to standard finite elements is that the quantities of interest in the interface elements are in the local coordinate system of the crack they represent. It is thus necessary to distinguish the upper and lower faces of the joint and work with the normal and tangential relative displacements at any point along the mid plane of the crack (Fig. 1).

Let us define the vector of relative displacements in a joint $\delta$ as the difference between the displacements at the upper and lower faces $\delta=\boldsymbol{u}_{\text {up }}-\boldsymbol{u}_{\text {low }}$. This vector is written in matrix form as:

$\delta=\left[\begin{array}{c}\delta_{x} \\ \delta_{y} \\ \delta_{z}\end{array}\right]=\left[\begin{array}{ccccccc}-N_{1} & 0 & 0 & \ldots & N_{n} & 0 & 0 \\ 0 & -N_{1} & 0 & \ldots & 0 & N_{n} & 0 \\ 0 & 0 & -N_{1} & \ldots & 0 & 0 & N_{n}\end{array}\right]\left[\begin{array}{c}u_{x 1} \\ u_{y 1} \\ u_{z 1} \\ \vdots \\ u_{x n} \\ u_{y n} \\ u_{z n}\end{array}\right]=\boldsymbol{N}_{u, I} \overline{\boldsymbol{u}}$

where $\overline{\boldsymbol{u}}$ is the standard vector of nodal displacements, and $\boldsymbol{N}_{u, I}$ is the matrix of shape functions of the interface element. Essentially, in the matrix $\boldsymbol{N}_{u, I}$, the shape functions $N_{i}$ are preceded by a negative sign for nodes located at the lower face of the joint and by a positive sign for nodes at the upper face.

Similarly as for beams or shells, we need to transform the relative displacements from the global to the local coordinate system. The transformation is written as:

$\check{\delta}=\left[\begin{array}{c}\delta_{l} \\ \delta_{m} \\ \delta_{n}\end{array}\right]=\left[\begin{array}{lll}\cos (\check{x}, x) & \cos (\check{x}, y) & \cos (\check{x}, z) \\ \cos (\check{y}, x) & \cos (\check{y}, y) & \cos (\check{y}, z) \\ \cos (\check{z}, x) & \cos (\check{z}, y) & \cos (\check{z}, z)\end{array}\right]\left[\begin{array}{c}\delta_{x} \\ \delta_{y} \\ \delta_{z}\end{array}\right]=\lambda \delta$

where $\lambda$ is the rotation matrix and $\check{x}, \breve{y}, \breve{z}$ are the local coordinate system at the interface (Fig. 1).

Following the formulation derived for the continuum solid-pore fluid mixture (Section 2), the behaviour of the interface elements is governed by two equilibrium equations. One equation deals with the mechanical response of the crack, whereas the other one describes the balance of fluid mass within the fracture. It is important to note that the formulation of the interface elements is completely compatible with the displacement-pore pressure formulation for the porous medium, and so 
both type of elements can be solved in the same coupled system of equations.

\subsubsection{Mechanical behaviour of the fracture}

The starting point is the strong form of the balance of momentum equation for the solid-fluid mixture at the fracture plane (Eq. (5)). We discretize the relative displacements at the joint and integrate the equation by parts to obtain the following weak form

$\boldsymbol{r}_{u, I}=\boldsymbol{M}_{I} \ddot{\overline{\boldsymbol{u}}}+\int_{\Omega_{I}} \boldsymbol{B}_{I}^{T} \sigma_{I}^{\prime} d \Omega_{I}-\boldsymbol{Q}_{I} \overline{\boldsymbol{p}}-\boldsymbol{f}_{u, I}=\mathbf{0}$

Eq. (31) shows the same structure as Eq. (10), but the different matrices and vectors are defined in the coordinate system in which the interface element is solved, i.e.:

$\boldsymbol{M}_{I}=\int_{\Omega_{I}} \boldsymbol{N}_{u, I}^{T} \rho \boldsymbol{N}_{u, I} d \Omega_{I} ; \quad \boldsymbol{Q}_{I}=\int_{\Omega_{I}} \boldsymbol{B}_{I}^{T} \alpha \boldsymbol{m}_{I} \boldsymbol{N}_{p} d \Omega_{I}$

$\boldsymbol{f}_{u, I}=\int_{\Omega_{I}} \boldsymbol{N}_{u, I}^{T} \rho \boldsymbol{b} d \Omega_{I}+\int_{\Gamma_{t}} \boldsymbol{N}_{u, I}^{T} \tilde{\boldsymbol{t}} d \Gamma_{I}$

where $\boldsymbol{B}_{I}$ is the deformation matrix of the interface element, defined as:

$\boldsymbol{B}_{I}=\frac{1}{t_{I}} \boldsymbol{N}_{u, I}$

with $t_{I}$ being the distance between the two faces of the interface (Fig. 1). If the interface is closed, then we use a small value $t_{I}=t_{\min }$ to avoid dividing by zero.

The effective stress vector in the fracture $\sigma_{I}^{\prime}$ must be computed first in the local coordinate system and then transformed into the global one as

$\sigma_{I}^{\prime}=\lambda^{T} \check{\sigma}_{I}^{\prime}=\left[\begin{array}{ccc}\cos (\check{x}, x) & \cos (\check{x}, y) & \cos (\check{x}, z) \\ \cos (\check{y}, x) & \cos (\check{y}, y) & \cos (\check{y}, z) \\ \cos (\check{z}, x) & \cos (\check{z}, y) & \cos (\check{z}, z)\end{array}\right]^{T}\left[\begin{array}{c}\sigma_{l}^{\prime} \\ \sigma_{m}^{\prime} \\ \sigma_{n}^{\prime}\end{array}\right]$

Also, vector $\boldsymbol{m}_{I}=\left[\begin{array}{lll}0, & 0,1\end{array}\right]^{T}$ comes from the definition of the local effective stresses in the joint as

$\check{\sigma}_{I}^{\prime}=\check{\sigma}_{I}+\alpha p \boldsymbol{m}_{I}=\left[\begin{array}{c}\sigma_{l} \\ \sigma_{m} \\ \sigma_{n}\end{array}\right]+\alpha p\left[\begin{array}{l}0 \\ 0 \\ 1\end{array}\right]$

The constitutive law governing the mechanical behaviour of the interface elements is a bilinear cohesive fracture model (Fig. 2) based on the fracture criteria of Camacho and Ortiz [28] and Song et al. [57].

Fig. 2a relates the normalized equivalent stress $\varsigma_{\text {eq,I }}$ with an internal state variable $\rho$ and shows that the evolution of the cohesive zone is an irreversible damage process. The variable $\rho$ plays the same role as the scalar variable $r(t)$ (Eq. (22)). It is defined as

$\rho(t)=\min \left\{\max \left\{\rho_{y}, \max _{\tau \leqslant t} \varepsilon_{e q, I}(\tau)\right\}, 1\right\}$

where $\rho_{y}$ is the damage threshold of the joints, and $\varepsilon_{e q, I}$ is the equivalent strain. Since the chosen fracture model is designed for mixed mode fracture, the evolution of the damage propagation in the cohesive fracture zone depends on the simultaneous activation of the tangential and normal relative displacements, i.e.

$\varepsilon_{e q, I}=\frac{\sqrt{\delta_{l}^{2}+\delta_{m}^{2}+\delta_{n}^{2}}}{\delta_{c}}$

with $\delta_{c}$ being the critical relative displacement, i.e. it is the relative displacement at which the cohesive zone stops transmitting forces.

Similarly, we can define the normalized equivalent stress at the interface as:

$\varsigma_{e q, I}=\frac{\sqrt{\left(\sigma_{l}^{\prime}\right)^{2}+\left(\sigma_{m}^{\prime}\right)^{2}+\left(\sigma_{n}^{\prime}\right)^{2}}}{\sigma_{y}}$

with $\sigma_{y}$ being the yield stress at the cohesive zone, i.e. the stress at which the cohesive zone starts damaging. Each local component of the stress is computed as follows:

$\sigma_{i}^{\prime}=\frac{\sigma_{y}(1-\varrho)}{\varrho \delta_{c}\left(1-\varrho_{y}\right)} \delta_{i} \quad i=l, m, n$

\subsubsection{Fluid flow in the fracture}

The fluid flow in the fracture is modelled by the mass balance equation:

$\boldsymbol{r}_{p, I}=\boldsymbol{Q}_{I}^{T} \dot{\boldsymbol{u}}+\boldsymbol{C}_{I} \dot{\overline{\boldsymbol{p}}}+\boldsymbol{H}_{I} \overline{\boldsymbol{p}}-\boldsymbol{f}_{p, I}=\mathbf{0}$

where

$\boldsymbol{C}_{I}=\int_{\Omega_{I}} \boldsymbol{N}_{p}^{T} \frac{1}{Q} \boldsymbol{N}_{p} d \Omega_{I} ; \quad \boldsymbol{H}_{I}=\int_{\Omega_{I}}\left(\nabla \boldsymbol{N}_{p}\right)_{I}^{T} \frac{1}{\mu} \boldsymbol{k}_{I}\left(\nabla \boldsymbol{N}_{p}\right)_{I} d \Omega_{I}$

$\boldsymbol{f}_{p, I}=\int_{\Omega_{I}}\left(\nabla \boldsymbol{N}_{p}\right)_{I}^{T} \frac{1}{\mu} \boldsymbol{k}_{I} \rho_{f} \boldsymbol{b} d \Omega_{I}-\int_{\Gamma_{q}} \boldsymbol{N}_{p}^{T} \widetilde{q}_{n} d \Gamma_{I}$

The pressure at the joint is interpolated as in the case of a continuum porous domain $p=\boldsymbol{N}_{p} \overline{\boldsymbol{p}}$. Hence, the compressibility matrix $\boldsymbol{C}_{I}$ in (42) is virtually the same as the one in (13), with the only difference being the domain of integration with $d \Omega_{I}=d A_{I} t_{I}$.

Matrix $\boldsymbol{H}_{I}$ introduces the enhanced permeability of the fractures in the porous medium. It is assumed that the longitudinal flow is coupled to the interface opening according to a cubic law [58,59], which is

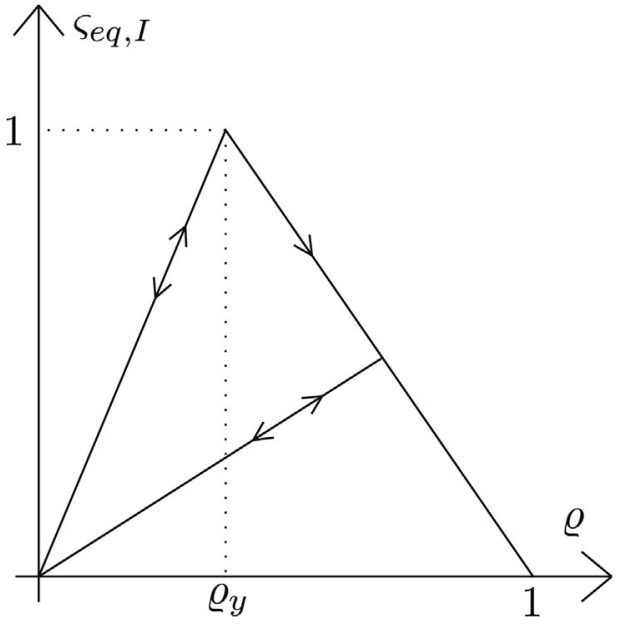

(a) Bilinear cohesive law.

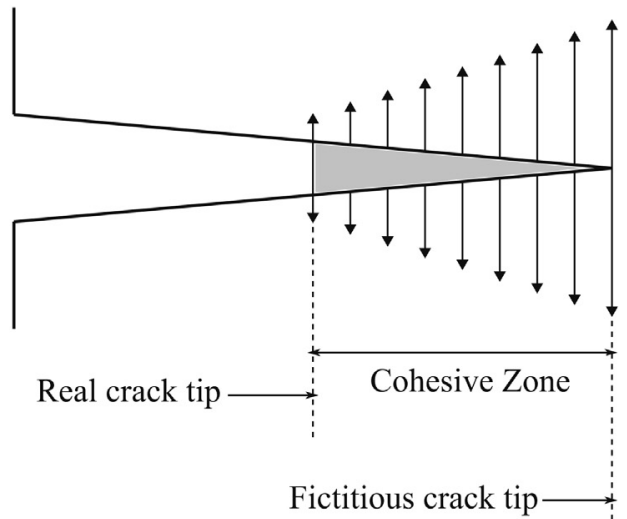

(b) Cohesive fracture zone. 
naturally incorporated in the gradient of the matrix of shape functions $\left(\nabla \boldsymbol{N}_{p}\right)_{I}$ and the intrinsic permeability matrix $\boldsymbol{k}_{I}$.

Matrix $\left(\nabla \boldsymbol{N}_{p}\right)_{I}$ is defined as [60]:

$\left(\nabla \boldsymbol{N}_{p}\right)_{I}=\left[\begin{array}{ccccc}\partial N_{1} / \partial l & \partial N_{2} / \partial l & \ldots & \partial N_{n-1} / \partial l & \partial N_{n} / \partial l \\ \partial N_{1} / \partial m & \partial N_{2} / \partial m & \ldots & \partial N_{n-1} / \partial m & \partial N_{n} / \partial m \\ -N_{1} / t_{I} & -N_{2} / t_{I} & \ldots & N_{n-1} / t_{I} & N_{n} / t_{I}\end{array}\right]$

The two first rows in (44) allow computing the derivatives of the pressure with respect to the tangential directions of the joint $l$ and $m$, whereas the third row approximates the derivative of the pressure in the normal direction $n$ by means of the pressure drop between the upper and lower faces of the interface.

Regarding the intrinsic permeability matrix of the fracture $\boldsymbol{k}_{I}$, we make a distinction between the permeability in the tangential directions from the permeability in the normal one, i.e.

$\boldsymbol{k}_{I}=\left[\begin{array}{ccc}k_{l} & 0 & 0 \\ 0 & k_{m} & 0 \\ 0 & 0 & k_{n}\end{array}\right]$

where $k_{l}$ and $k_{m}$ are defined as [58]:

$k_{l}=k_{m}=\frac{t_{I}^{2}}{12}$

The parameter $k_{n}$ represents the transversal permeability of the fracture, which is usually given a value similar to the intrinsic permeability of the porous domain.

\subsection{Fracture propagation approach}

The fracture propagation strategy is based on the insertion of new interface elements where the strength of the porous domain reaches a threshold value $[61,62]$. This method provides a realistic approach for the simulation of dynamic crack propagation, but it requires remeshing the potential fracture zone after the insertion of every new interface element.

As mentioned in the introduction, we essentially combine the integral-type non-local damage model of Section 3.1 with the presented quasi-zero-thickness interface elements. The fundamental idea is to use the information of the damaged points around the crack tip to determine, as accurately as possible, the direction of the fracture propagation. The method relies on two main assumptions:

- Any crack growth process must start from a predefined crack tip.

- Such propagation can either follow one direction, or bifurcate in two.
The first assumption implies that the implemented method cannot model crack initiation. However, this is not major inconvenience because one can always use the damage model to determine the initial damage pattern, and then manually insert crack tips to model fracture propagation. The second assumption means that the presented approach allows capturing crack branching for any propagation process.

Next, we present the main steps of the crack propagation technique.

\subsubsection{Crack tip neighbours search}

The information of the damaged elements around the crack tip is used to estimate the direction of the crack path. In order to do so, a neighbour search must be performed at the beginning of each time step.

Let $l_{p}$ be the propagation length, a material parameter determining the domain of influence of the fracture $\Omega_{f}$. For each fracture tip, we search the neighbouring Gauss points falling inside a circle (or a sphere in 3D) of radius $l_{p}$ (Fig. 3a).

At this stage we also divide the domain of influence $\Omega_{f}$ into quarters and distribute the neighbour points between the Top-Front-Quarter (TFQ) and the Bottom-Front-Quarter (BFQ) (Fig. 3b).

\subsubsection{Crack growth}

In order to determine the start of the crack growth, we estimate the damage at the crack tip $\mathbb{d}_{t}$ and compare it with a prefixed threshold value, called propagation damage $\mathbb{d}_{p}$.

Damage at the crack tip is defined here as a non-local measure of the damage inside the region of influence of the fracture $\Omega_{f}$. This measure follows the same idea as the non-local average performed in (26). Damage is evaluated as:

$\mathrm{d}_{t}=\sum_{q} Z_{t q} \mathrm{~d}_{q} w_{q} \quad \forall q \in \Omega_{f}$

where $w_{q}$ is a coefficient containing the product of the determinant of the Jacobian and the integration weight of Gauss point $q$, and $Z_{t q}$ is the weight of non-local interaction between the tip and any other point $q$, defined as in Eq. (27), i.e.

$Z_{t q}=\frac{Z_{0}\left(\mathscr{D}_{t q}\right)}{\sum_{r} Z_{0}\left(\mathscr{D}_{t r}\right) w_{r}}$

When the damage at the tip exceeds the threshold value $\left(\mathbb{d}_{t}>\mathbb{d}_{p}\right)$ the crack is supposed to grow, but it is still necessary to discern the direction of propagation. First, we compute the potential location for the new crack tip by weighted averaging the coordinates of all the points in front of the crack tip. Unlike the average performed in (47), the coordinates are weighted in terms of the damage of each Gauss

\begin{tabular}{|c|c|c|c|c|}
\hline $\begin{array}{l}\times \quad \times \\
\times{ }^{\times} \times\end{array}$ & ${ }_{\times}^{x} \times x$ & $\times$ & $\operatorname{x}_{x}^{x} x^{\stackrel{x}{x}}$ & $\begin{array}{l}x^{x} \times \\
x_{x}^{x} \times\end{array}$ \\
\hline $\begin{array}{c}\times \quad \times \\
\times \quad \times \\
\times \quad \times\end{array}$ & $\begin{array}{ccc}x^{x} & x & x \\
\times & x_{x}^{x}\end{array}$ & $\times \stackrel{x}{\times} \times$ & $\begin{array}{cc}\times & \times \\
\times & \times \\
\times & \times \\
& \times\end{array}$ & $\begin{array}{l}x^{x} \times \\
\times x\end{array}$ \\
\hline$x_{x} \times$ & & $\begin{array}{l:l}x & x \\
x & x \\
x & x \\
x & x \\
x & x \\
x & x\end{array}$ & $\begin{array}{l}x_{x} \\
x_{x}^{x} \\
x\end{array}$ & $x^{x \times} \times$ \\
\hline $\begin{array}{cr}\times & \times \\
\times & \times \\
\times & \end{array}$ & $\begin{array}{ll}\times & \times \\
\times & \times \\
\times & \times \\
& \\
& \times\end{array}$ & $\begin{array}{r}\times{ }^{x} \\
\times \times\end{array}$ & $\times x_{x}^{x^{x}}$ & $\begin{array}{ll} & \times \\
\times & \times \\
\times & \times \\
& \end{array}$ \\
\hline $\begin{array}{r}\times \times \\
\times \quad \times\end{array}$ & $\begin{array}{l}x \\
x^{x} \\
x\end{array}$ & $\begin{array}{cc}\times & \times \\
\times & \times \\
\times & \times\end{array}$ & $\mid \begin{array}{ll}x & x^{x} \\
\times & x^{x} x \\
& x^{x}\end{array}$ & $\begin{array}{l}\times \times{ }^{x} \\
\times \quad \times\end{array}$ \\
\hline
\end{tabular}

(a) Grid-based search.
Fig. 3. Crack tip neighbours search. (b) Division of the domain of influence into quarters. 
point under consideration. In essence, the coordinate of the new crack tip $\boldsymbol{x}_{t}$ is computed as:

$\boldsymbol{x}_{t}=\sum_{q} \widehat{Z}_{t q} \boldsymbol{x}_{q} w_{q} \quad \forall q \in T F Q \cup B F Q$

where $\widehat{Z}_{t q}$ is a modified version of the weighting function in (48) as

$\widehat{Z}_{t q}=\frac{\mathrm{d}_{q}}{\sum_{r} \mathrm{~d}_{r} w_{r}}$

Once the coordinates for the new crack tip have been found, it is necessary to check whether it is at a feasible location of the domain. Basically, the new crack tip must fulfil the following two conditions:

- It must fall inside an existing element in the mesh.

- The average damage at the element must be larger than a minimum value $\mathbb{d}_{e} \geqslant \mathbb{d}_{\text {min }}$

The first condition is obviously necessary to avoid propagating the fracture outside the limits of the model, whereas the second condition prevents propagating the fracture towards undamaged regions.

In case one of the two conditions is not fulfilled, we recalculate two possible coordinates for the crack tip, distinguishing the elements of the top-front-quarter (TFQ) and the bottom-front-quarter (BFQ) as

$\begin{array}{ll}\boldsymbol{x}_{t_{T}}=\sum_{q} \widehat{Z}_{t q} \boldsymbol{x}_{q} w_{q} & \forall q \in T F Q \\ \boldsymbol{x}_{t_{B}}=\sum_{q} \widehat{Z}_{t q} \boldsymbol{x}_{q} w_{q} \quad \forall q \in B F Q\end{array}$

Of course, the two coordinates in (51) must also fulfil the aforementioned conditions to make sure they are feasible crack tips. In this regard, three different scenarios are possible. If the two new tips are valid, then the fracture bifurcates in two separate ways. If only one of the computed coordinates is feasible, then the fracture will propagate only towards that valid tip. Finally, if neither of the tips is valid, the fracture does not grow.

\subsubsection{Insertion and remeshing}

After estimating the direction of the propagating fracture, new interface elements are inserted into the model, ensuring that the new generated mesh is conformal. We use the pre and post-processor GiD for that purpose [63].

Thereby, not only GiD meshes the geometry at the beginning of the numerical simulation, but it is also GiD which generates the new spatial discretization every time we need to adapt the model with new interface elements. In this work, a uniform mesh is generated for simplicity, but one could also apply the method proposed in [64] for adapting the mesh of the whole domain in a non-structured manner according to the stress state.

After the new model is generated, a final step must be performed: the mapping of primary unknowns and internal variables between the old and new meshes. The mapping of the primary unknowns, i.e. nodal displacements and nodal pore pressure, is carried out by using shape function projections, whereas the mapping of internal state variables, i.e. variables $r$ and $\varrho$, is performed through a weighted spatial averaging. This procedure is similar to the non-local average performed in (26) with the difference that, in this case, the source points are the Gauss points of the old mesh and the receiver points are the integration points of the new mesh. In order to ensure a consistent damage field in the transition between the pre and post insertion of interface elements, the internal state variable $\rho$ of each new element is defined as the maximum value between the computed average damage and the propagation damage $\mathbb{d}_{p}$.

\section{Examples}

This section includes three test cases devoted to assess the performance of the presented fracture propagation strategy and the behaviour of the implemented interface elements. The first example is meant to validate the fracture propagation approach against an analytical solution, whereas the second and third cases are used to test the proposed technique under complex conditions and evaluate its strengths and limitations.

The three examples model fluid-driven fracture propagation problems in nearly undrained-incompressible porous media. In all cases we use FIC-stabilized elements of equal order interpolation for the displacements and the pore pressure. The two first problems are analysed in a $2 \mathrm{D}$ framework under plane strain conditions, and the last test is solved with a 3D model. In all cases, the porous medium is considered to have isotropic permeability and the effect of gravity is not considered.

\subsection{Fluid-driven fracture propagation test}

This example consists on a semi-cylindrical portion of rock of $80 \mathrm{~m}$ radius, laying on a rectangle $10 \mathrm{~m}$ high that has a notch of $5 \times 50 \mathrm{~cm}$ at the center. Water is injected at a constant rate of $q_{i n}=0.2 \mathrm{~m} / \mathrm{s}$ through an incipient crack tip of $0.5 \mathrm{~mm}$ thick and $125 \mathrm{~mm}$ long, which results in a volumetric flow of $1 \cdot 10^{-4} \mathrm{~m}^{3} / \mathrm{s}$. The geometry and boundary conditions of the problem are shown in Fig. 4.

The material of the porous domain is considered to undergo isotropic degradation by means of the damage model defined in Section 3.1 , whereas the predefined crack tip is represented by quasi-zerothickness interface elements. The material properties are summarized in Table 1.

As commented above, this problem is approached in a $2 \mathrm{D}$ configuration under plane-strain assumption. The porous domain is solved with the FIC-stabilized formulation, using 3-noded triangular elements with linear interpolation for the displacements and the pore pressure. The interface elements of the crack tip are 4-noded quadrilateral elements.

The main objective of this test is to validate the implemented propagation technique against an analytical solution obtained by Spence and Sharp [65] and replicated by Khoei et al. [60]. The fluid pressure along the crack mouth, the crack length and the crack width have been monitored through $10 \mathrm{~s}$ of simulation. In order to assess the robustness of the numerical solution, a convergence analysis was performed in terms of the mesh size and the time step.

The influence of the mesh size was analysed by fixing the time step at $\Delta t=0.02 \mathrm{~s}$ and solving the problem for three different spatial discretizations (Fig. 5). The first model was obtained from a characteristic element size of $l_{e}=4 \mathrm{~cm}$, with a resultant mesh of 15,330 elements and

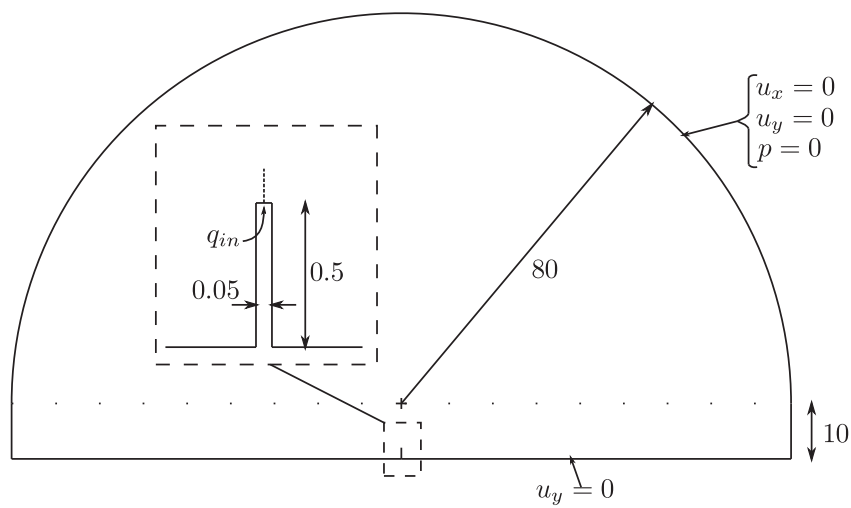

Fig. 4. Fluid-driven fracture propagation test. Geometry and conditions. Dimensions in $m$. 
Table 1

Fluid-driven fracture propagation test. Material properties.

\begin{tabular}{|c|c|c|c|}
\hline Property & Rock & Joints & Units \\
\hline Young's modulus $(E)$ & $1.596 \cdot 10^{10}$ & $1.596 \cdot 10^{10}$ & $\mathrm{~N} / \mathrm{m}^{2}$ \\
\hline Poisson's ratio $(\nu)$ & 0.33 & 0.33 & - \\
\hline Solid density $\left(\rho_{S}\right)$ & 2000 & 2000 & $\mathrm{~kg} / \mathrm{m}^{3}$ \\
\hline Fluid density $\left(\rho_{f}\right)$ & 1000 & 1000 & $\mathrm{~kg} / \mathrm{m}^{3}$ \\
\hline Porosity $(\phi)$ & 0.19 & 0.19 & - \\
\hline Solid bulk modulus $\left(K_{S}\right)$ & $3.6 \cdot 10^{10}$ & $3.6 \cdot 10^{10}$ & $\mathrm{~N} / \mathrm{m}^{2}$ \\
\hline Fluid bulk modulus $\left(K_{f}\right)$ & $3 \cdot 10^{9}$ & $3 \cdot 10^{9}$ & $\mathrm{~N} / \mathrm{m}^{2}$ \\
\hline Intrinsic permeability $\left(k / k_{n}\right)$ & $6 \cdot 10^{-15}$ & $6 \cdot 10^{-15}$ & $\mathrm{~m}^{2}$ \\
\hline Dynamic viscosity $(\mu)$ & 0.001 & 0.001 & $\mathrm{~N} / \mathrm{m}^{2} \cdot \mathrm{s}$ \\
\hline Strength ratio $(\kappa)$ & 25 & - & - \\
\hline Residual strength $(R)$ & 0.95 & - & - \\
\hline Softening slope $(S)$ & $1.05 \cdot 10^{4}$ & - & - \\
\hline Damage threshold $\left(r_{y} / \varsigma_{y}\right)$ & $1.5 \cdot 10^{-4}$ & $1 \cdot 10^{-4}$ & - \\
\hline Minimum joint width $\left(t_{\min }\right)$ & - & $1 \cdot 10^{-4}$ & $\mathrm{~m}$ \\
\hline Critical displacement $\left(\delta_{c}\right)$ & - & 0.01 & $\mathrm{~m}$ \\
\hline Yield stress $\left(\sigma_{y}\right)$ & - & $7 \cdot 10^{6}$ & $\mathrm{~N} / \mathrm{m}^{2}$ \\
\hline Friction coefficient $\left(\mu_{F}\right)$ & - & 0.4 & - \\
\hline Characteristic length $\left(l_{c}\right)$ & 0.1 & - & $\mathrm{m}$ \\
\hline Propagation length $\left(l_{p}\right)$ & - & 0.06 & $\mathrm{~m}$ \\
\hline Propagation damage $\left(\mathbb{d}_{p}\right)$ & - & 0.6 & - \\
\hline
\end{tabular}

7846 nodes. The second mesh, with 44,708 elements and 22,580 nodes, resulted from an element size of $l_{e}=2 \mathrm{~cm}$. The third model was obtained after defining a characteristic element size of $l_{e}=1 \mathrm{~cm}$ and lead to a mesh of 153,582 elements and 77,095 nodes.

In order to study the effect of the time step on the solution, we fixed the mesh size at $l_{e}=2 \mathrm{~cm}$ and run the case for three different time steps: $\Delta t=0.04 \mathrm{~s}, \Delta t=0.02 \mathrm{~s}$ and $\Delta t=0.01 \mathrm{~s}$.
Fig. 6a, c and e show the pressure, crack length and crack width for different mesh size and $\Delta t=0.02 \mathrm{~s}$. The results obtained agree with the analytical solutions. We highlight that the combination of an integraltype non-local damage model with the insertion of interface elements has no mesh dependence.

The results displayed in Fig. $6 \mathrm{~b}, \mathrm{~d}$ and $\mathrm{f}$ evidence that the time step is an important parameter in this kind of problems. Indeed, despite integrating the time variable with an unconditionally stable Newmark scheme, a large time step together with the material non-linearity of the problem lead to inaccurate results. Such effect is particularly remarkable in Fig. $6 \mathrm{f}$ mainly because of the greater sensitivity of the crack width variable (in the range of millimetres) with respect to the crack length and the pore pressure variables (in the range of meters and mega pascals, respectively). In any case, using a small enough time step ensures obtaining consistent results. In this case good results have been obtained for $\Delta t \leqslant 0.02 \mathrm{~s}$.

Regarding the validation of the present test, a general good agreement is observed between the numerical and the analytical curves. Looking at Fig. 6a and b, it is evident that the pressure at the mouth falls faster in the beginning of the numerical solutions. Such pressure drop is related to a sudden lost of integrity of the material, and thus an accurate calibration of the material properties could smooth this effect. However, after a few seconds all curves converge to the same value of $0.5 \mathrm{MPa}$, which remains virtually constant due to the near undrained condition of the rock. In Fig. $6 \mathrm{c}$ and d, one observes that the computed crack grows at a slightly slower pace than the expected solution. This fact is clearly linked to the fast drop in the pressure already commented. Finally, it is noticeable the similarity between the theoretical crack width and the numerical one. Except for the solution with $\Delta t=0.04 \mathrm{~s}$, the slope of the curve and the maximum value reached in the rest of the cases are virtually identical to the analytical result.

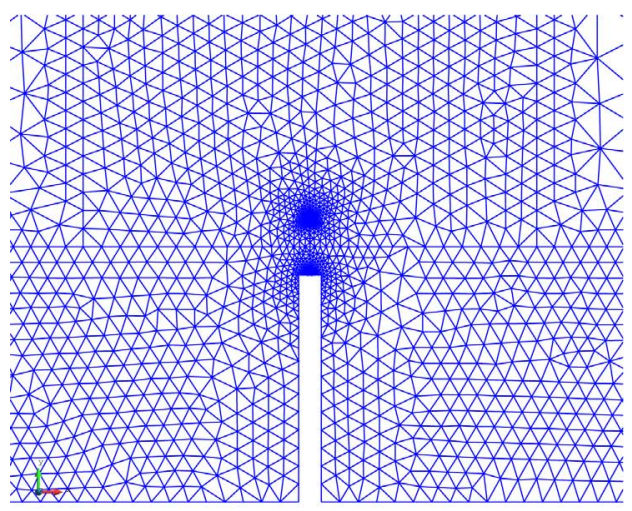

(a) $l_{e}=4 \mathrm{~cm}: 15,330$ elements.

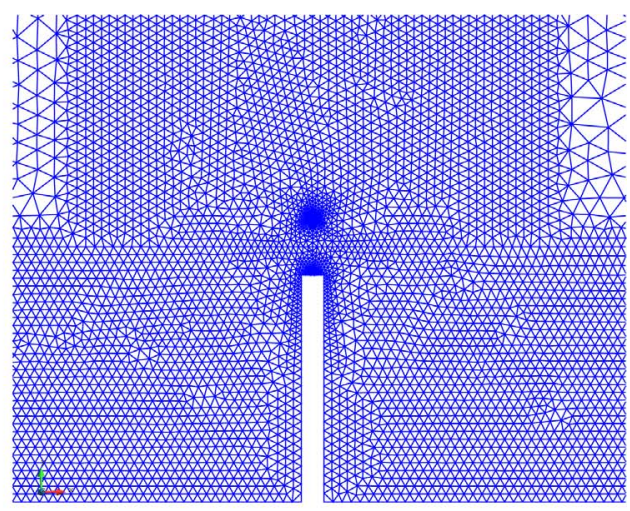

(b) $l_{e}=2 \mathrm{~cm}: 44,708$ elements.
Fig. 5. Fluid-driven fracture propagation test. Initial meshes.

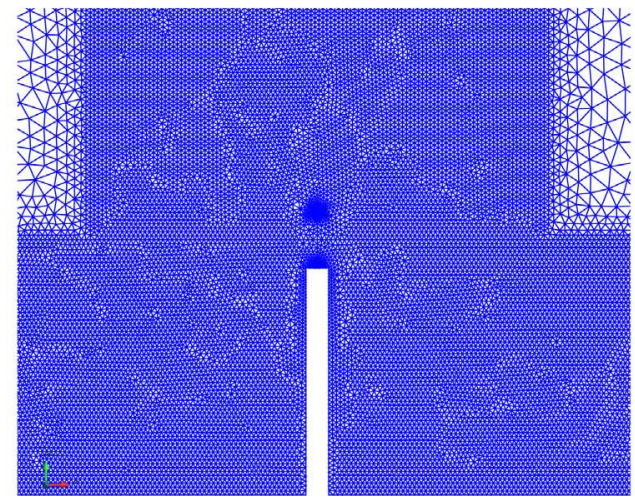

(c) $l_{e}=1 \mathrm{~cm}: 153,582$ elements. 


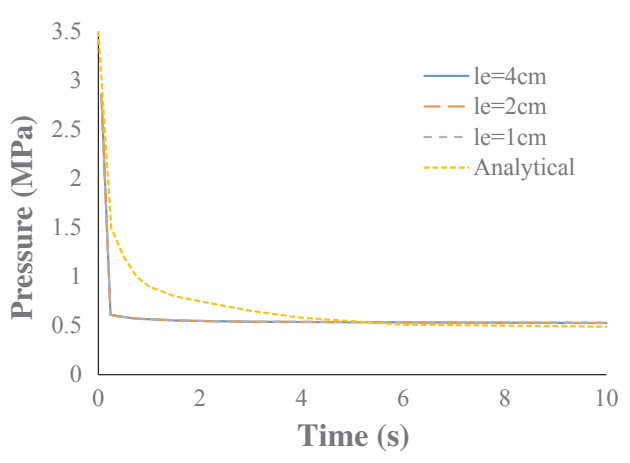

(a) Pressure for different mesh sizes with $\Delta t=0.02 \mathrm{~s}$.

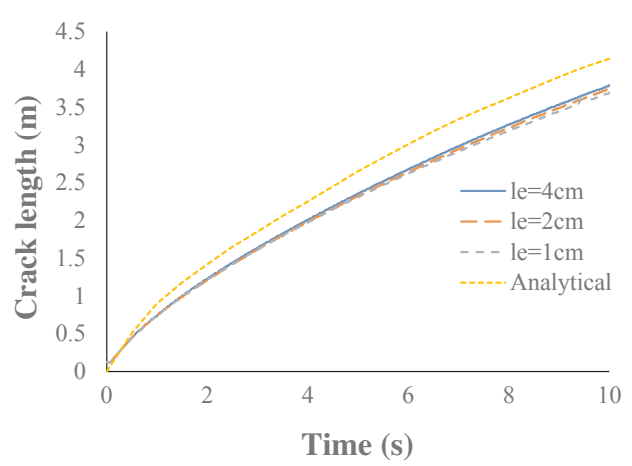

(c) Crack length for different mesh sizes with $\Delta t=0.02 \mathrm{~s}$.

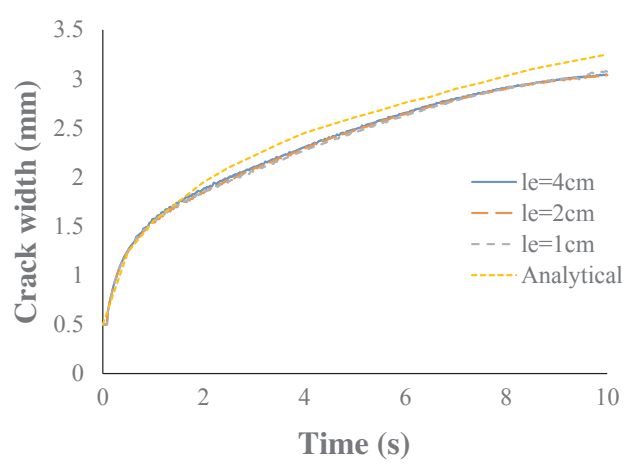

(e) Crack width for different mesh sizes with $\Delta t=0.02 \mathrm{~s}$.

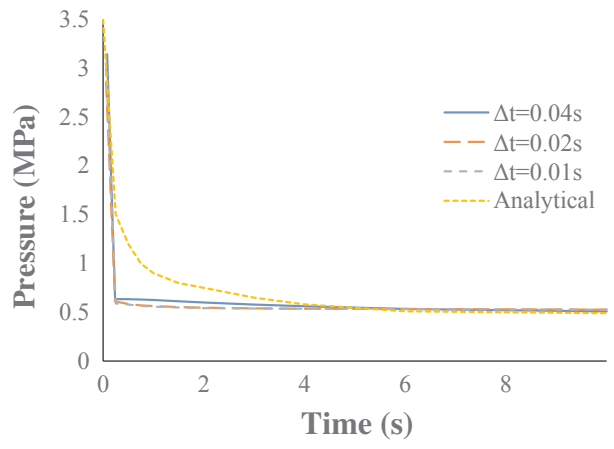

(b) Pressure for different time steps with $l_{\mathrm{e}}=2 \mathrm{~cm}$.

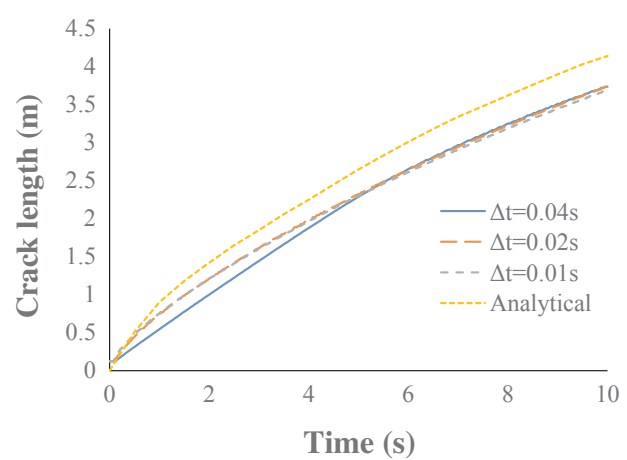

(d) Crack length for different time steps with $l_{\mathrm{e}}=2 \mathrm{~cm}$.

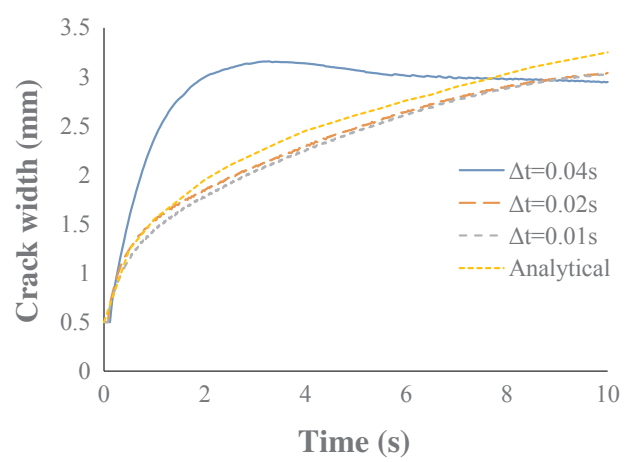

(f) Crack width for different time steps with $l_{\mathrm{e}}=2 \mathrm{~cm}$.
Fig. 6. Fluid-driven fracture propagation test. Time evolution of the pressure, the crack length and the crack width.

\subsection{Crack tracking test}

This second test case models a rectangular saturated porous domain of $6 \times 5 \mathrm{~m}$. The same notch and crack tip of the previous example have been horizontally placed at the left side of the model, with equivalent conditions at the inlet. An incursion of stronger rock is defined over $60 \mathrm{~cm}$ apart from the initial crack tip, as represented in Fig. 7.

The porous media and the crack tip are characterized with the same properties of the previous example (Table 1). The only difference is in the material incursion in front of the crack tip (see Table 2).

Again, this case is solved as a plane-strain 2D problem, and FICstabilized 3-noded triangles are used to circumvent the violation of Babuska-Brezzi conditions due to the impermeability of the porous medium. Also, in the light of the results in the last example, the characteristic element size chosen is $l_{e}=2 \mathrm{~cm}$ and $\Delta t=0.02 \mathrm{~s}$.

The main purpose of the present test is to verify the crack-tracking capabilities of the implemented approach against anisotropic porous domains. In order to do so, three different "obstacles" have been placed

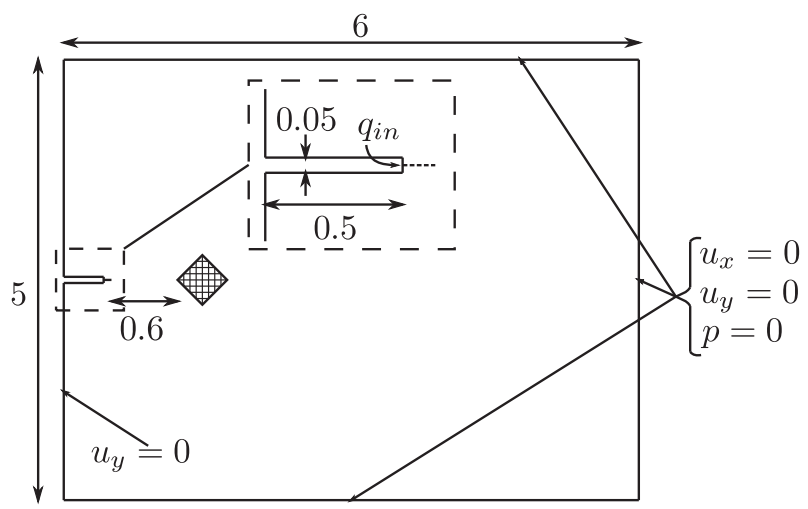

Fig. 7. Crack tracking test. Geometry and conditions. Dimensions in $m$. 
Table 2

Crack tracking test. Material properties of the incursion.

\begin{tabular}{lcr}
\hline Property & Incursion & Units \\
\hline Young's modulus $(E)$ & $4.0 \cdot 10^{10}$ & $\mathrm{~N} / \mathrm{m}^{2}$ \\
Poisson's ratio $(\nu)$ & 0.33 & - \\
Solid density $\left(\rho_{s}\right)$ & 2000 & $\mathrm{~kg} / \mathrm{m}^{3}$ \\
Fluid density $\left(\rho_{f}\right)$ & 1000 & $\mathrm{~kg} / \mathrm{m}^{3}$ \\
Porosity $(\phi)$ & 0.19 & - \\
Solid bulk modulus $\left(K_{S}\right)$ & $3.6 \cdot 10^{10}$ & $\mathrm{~N} / \mathrm{m}^{2}$ \\
Fluid bulk modulus $\left(K_{f}\right)$ & $3 \cdot 10^{9}$ & $\mathrm{~N} / \mathrm{m}^{2}$ \\
Intrinsic permeability $(k)$ & $6 \cdot 10^{-15}$ & $\mathrm{~m}$ \\
Dynamic viscosity $(\mu)$ & 0.001 & $\mathrm{~N} / \mathrm{m}^{2} \cdot \mathrm{s}$ \\
Strength ratio $(\kappa)$ & 25 & - \\
Residual strength $(R)$ & 0.95 & - \\
Softening slope $(S)$ & $1.05 \cdot 10^{4}$ & - \\
Damage threshold $\left(r_{y}\right)$ & $5.5 \cdot 10^{-3}$ & $\mathrm{~m}$ \\
Characteristic length $\left(l_{c}\right)$ & 0.1 & \\
\hline
\end{tabular}

in front of a propagating crack (Fig. 8). The first two incursions (Fig. 8a and b) are meant to simulate branching situations for different bifurcation angles, whereas the third obstacle introduces an irregular preferential path for the advancing fracture (Fig. 8c).

The three crack paths, after $2 \mathrm{~s}$ of simulation, are displayed in Fig. 9. The fluid pressure and the damage of the porous domain are also illustrated to give a deeper insight into the current analysis.

Focusing first on Fig. 9a, c and e, it is evident that the proposed technique captures the anisotropy introduced in the model, and the inserted interface elements properly represent the enhanced permeability of the porous medium. Moreover, the smooth contour lines of the pressure field show that the FIC-stabilized 3-noded triangles have

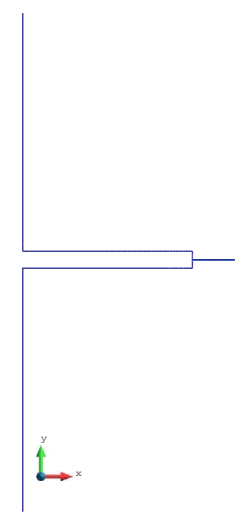

(a) Obstacle 1: 45-90-45 isosceles triangle.
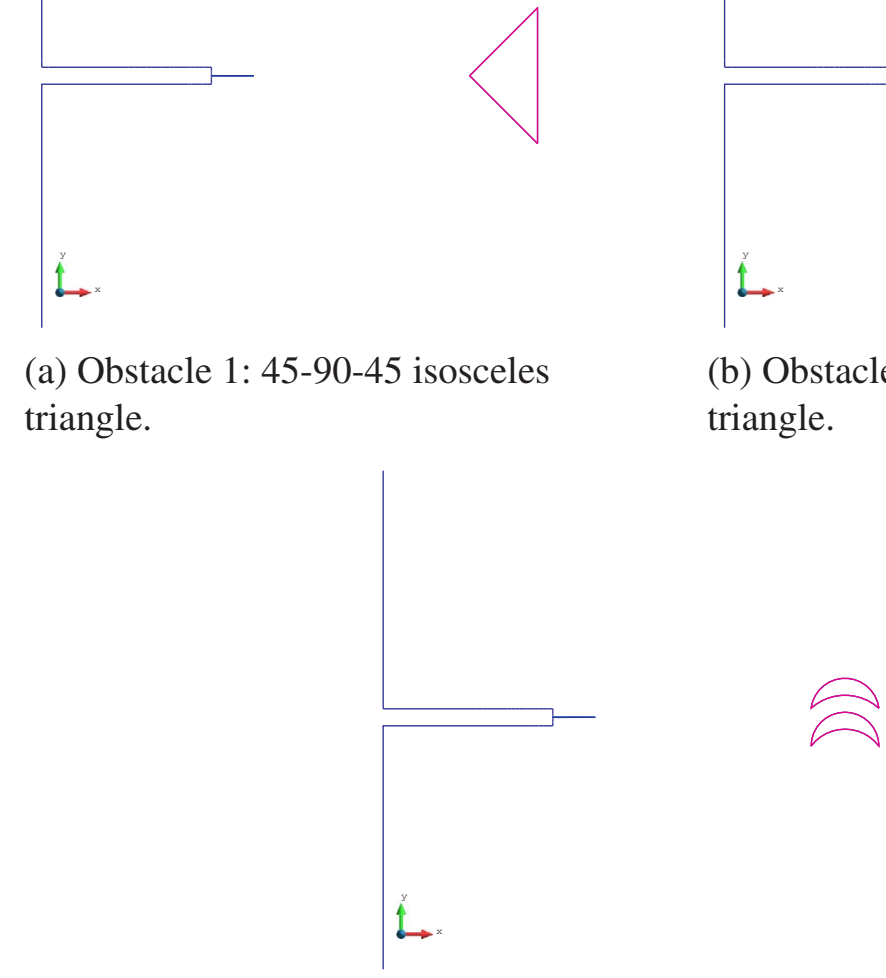

(c) Obstacle 3: moon shape incursions.

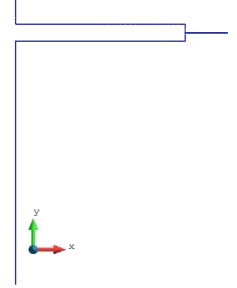

an excellent behaviour near the undrained-incompressible conditions. It is interesting to note that the maximum water pressure is different for each case, with the highest value in Fig. 9e and the lowest in Fig. 9a. Although the difference is subtle, one may infer that the pressure dissipates faster in a branching case with a wider angle of bifurcation.

In Fig. 9b, $d$ and $f$, the non-locality of the damage model is clearly visible in the width of the damage mark around the crack. Indeed, with a characteristic length of $l_{c}=0.1 \mathrm{~m}$, the crack tip is surrounded by a damaged area with a diameter of over $0.2 \mathrm{~m}$. Such diffusive damage pattern is what leads the crack through the right path, avoiding meshinduced directional bias. Thereby, in the first two cases (Fig. 9b and d) the fracture eventually runs into the stronger incursion and, given the low degradation of the material in front of the tip, the crack bifurcates with an angle that depends on the shape of the incursion. For the moon shape obstacles (Fig. 9f), the undamaged areas on both sides of the tip let the crack no choice but to pass between the two obstacles. A detailed view of the mesh is shown in Fig. 10.

Regarding the results in Fig. 9, it is also interesting to note the relation between damage and seepage in the proposed model. Indeed, the reduction of stiffness, given by the propagation of damage around the crack tip, introduces a gradient of pressure that contributes to the seepage of fluid from the fracture to the rest of the domain. This is shown by the green contour lines of the pressure field occupying all the damaged region.

\subsection{Parallel fracture propagation test}

This last example consists on a $4 \times 4 \times 6 \mathrm{~m}$ block of soil with two parallel crack tips separated by a distance $s$. Water is injected at a constant rate of $q_{i n}=6 \mathrm{~m} / \mathrm{s}$ through each one of the cracks of $0.5 \mathrm{~mm}$ thick and $125 \mathrm{~mm}$ long, giving a total volumetric flow of around

Fig. 8. Crack tracking test. Material incursions in front of the crack tip. (b) Obstacle 2: 75-30-75 isosceles triangle.

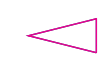




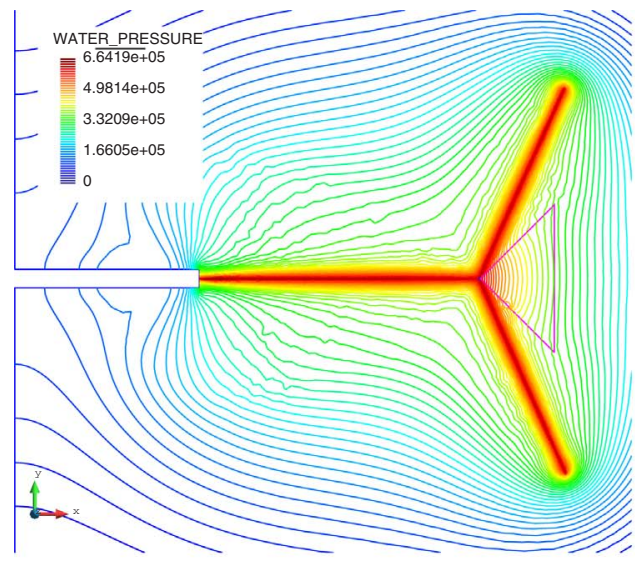

(a) Pressure with obstacle 1 .

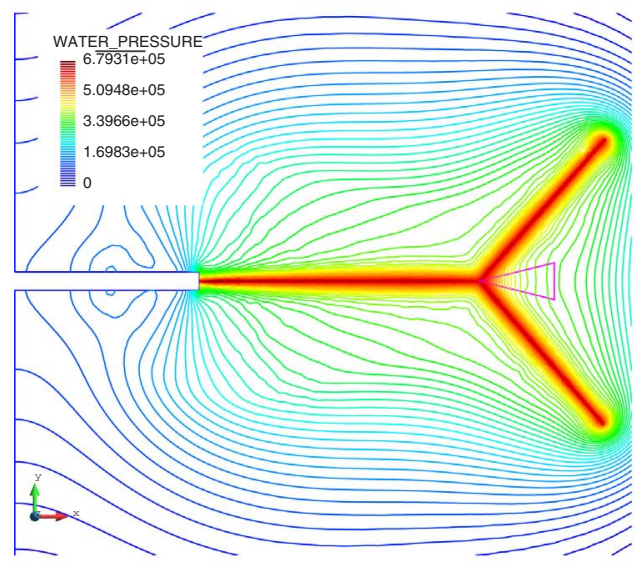

(c) Pressure with obstacle 2 .

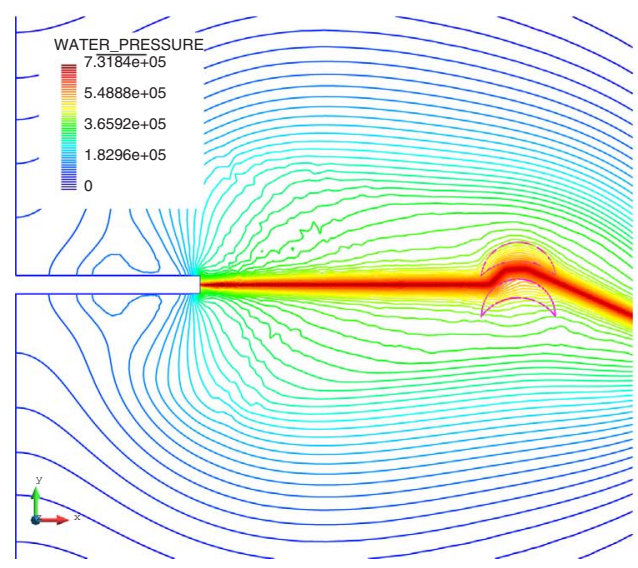

(e) Pressure with obstacle 3 .

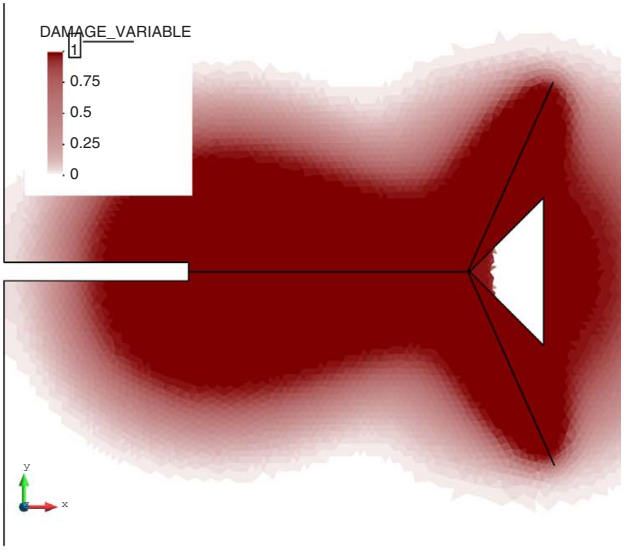

(b) Damage with obstacle 1 .

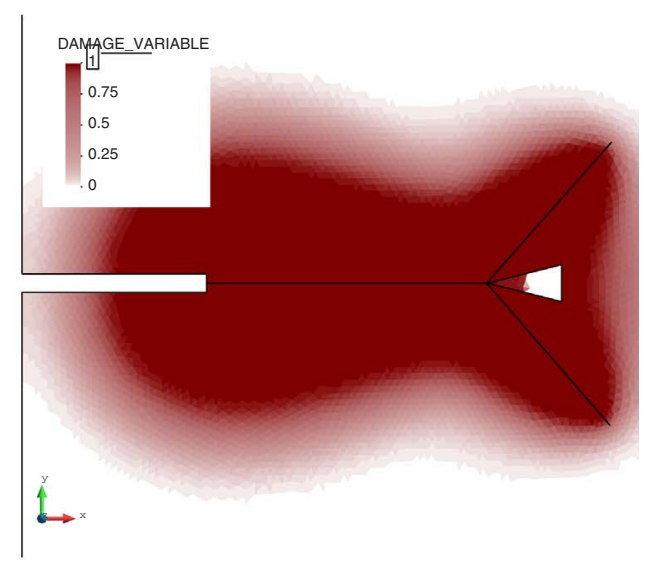

(d) Damage with obstacle 2 .

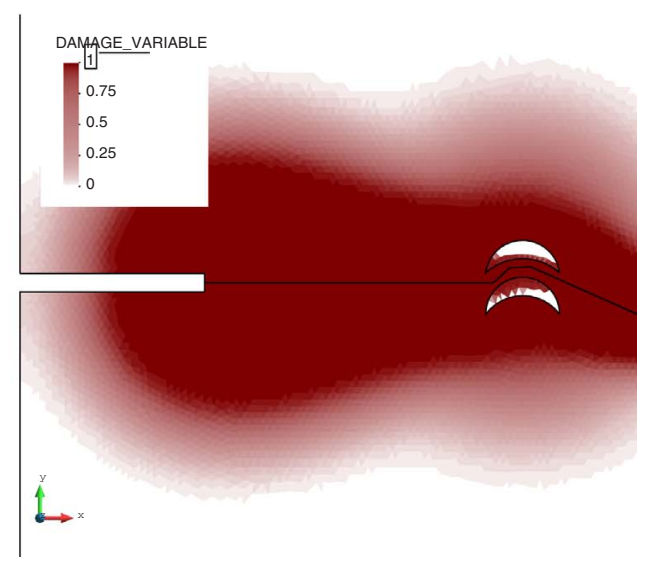

(f) Damage with obstacle 3 .

Fig. 9. Crack tracking test. Pore pressure and damage at $t=2 \mathrm{~s}$.

$1.5 \cdot 10^{-6} \mathrm{~m}^{3} / \mathrm{s}$. A scheme of the geometry and the boundary conditions is represented in Fig. 11.

In this case a softer material has been defined in order to reduce the maximum pore pressure and minimize the computational cost. In this case, the characteristic element size and the time step are also larger than in previous examples with $l_{e}=5 \mathrm{~cm}$ and $\Delta t=0.05 \mathrm{~s}$. The material properties of the porous domain and the two incipient cracks are given in Table 3.

Since the problem is approached in a 3D framework, the porous domain is represented by stabilized 4-noded tetrahedra with linear interpolation for the displacements and the pore pressure. The two crack tips are meshed with six-node wedge interface elements. Also, it is important to note the different definition of the cracks in the present 3D case when compared to the previous 2D examples. Indeed, while the two-dimensional crack tip was defined from just three points (Fig. 12a), the three-dimensional fracture tip is assumed to be conical, but it is approximated as a pyramid of quadrilateral base (Fig. 12b).

Basically, the purpose of this problem is to analyse the influence of the distance between neighbouring cracks in a parallel fracture propagation case. Thereby, in order to give a more realistic insight and 


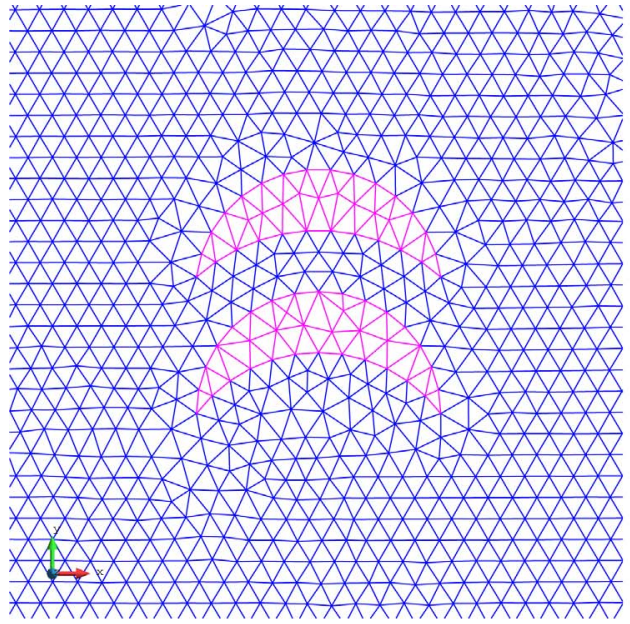

(a) Mesh at initial stage.

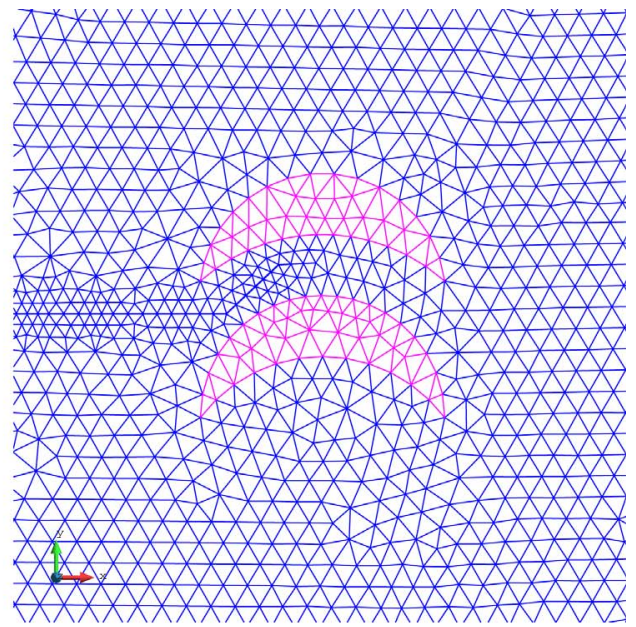

(b) Mesh at intermediate stage.
Fig. 10. Crack tracking test. Fracture passing between moon shape incursions.

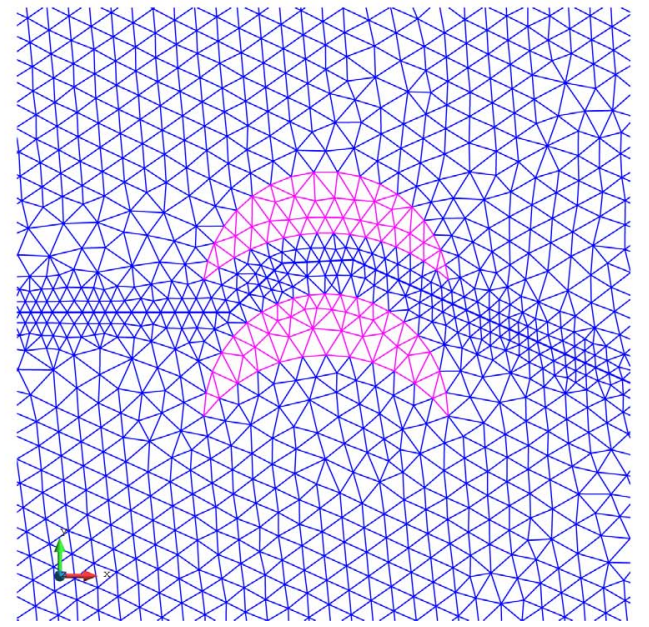

(c) Mesh at advanced stage.

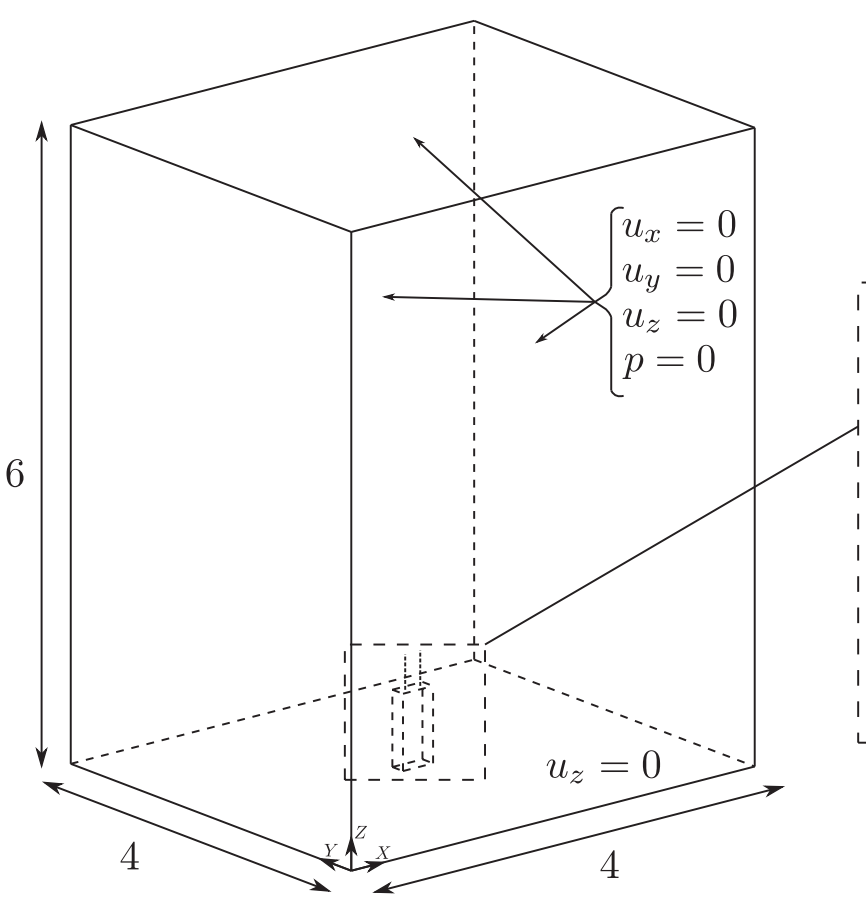

Fig. 11. Parallel fracture propagation test. Geometry and conditions. Dimensions in $m$. 
Table 3

Parallel fracture propagation test. Material properties.

\begin{tabular}{|c|c|c|c|}
\hline Property & Soil & Joints & Units \\
\hline Young's modulus ( $E$ ) & $2 \cdot 10^{9}$ & $2 \cdot 10^{9}$ & $\mathrm{~N} / \mathrm{m}^{2}$ \\
\hline Poisson's ratio $(\nu)$ & 0.1 & 0.1 & - \\
\hline Solid density $\left(\rho_{S}\right)$ & 2000 & 2000 & $\mathrm{~kg} / \mathrm{m}^{3}$ \\
\hline Fluid density $\left(\rho_{f}\right)$ & 1000 & 1000 & $\mathrm{~kg} / \mathrm{m}^{3}$ \\
\hline Porosity $(\phi)$ & 0.3 & 0.3 & - \\
\hline Solid bulk modulus $\left(K_{S}\right)$ & $1.5 \cdot 10^{17}$ & $1.5 \cdot 10^{17}$ & $\mathrm{~N} / \mathrm{m}^{2}$ \\
\hline Fluid bulk modulus $\left(K_{f}\right)$ & $3 \cdot 10^{14}$ & $3 \cdot 10^{14}$ & $\mathrm{~N} / \mathrm{m}^{2}$ \\
\hline Intrinsic permeability $\left(k / k_{n}\right)$ & $1 \cdot 10^{-14}$ & $1 \cdot 10^{-14}$ & $\mathrm{~m}^{2}$ \\
\hline Dynamic viscosity $(\mu)$ & 0.001 & 0.001 & $\mathrm{~N} / \mathrm{m}^{2} \cdot \mathrm{s}$ \\
\hline Strength ratio $(\kappa)$ & 10 & - & - \\
\hline Residual strength $(R)$ & 0.4 & - & - \\
\hline Softening slope $(S)$ & $1.5 \cdot 10^{4}$ & - & - \\
\hline Damage threshold $\left(r_{y} / \varrho_{y}\right)$ & $7.5 \cdot 10^{-6}$ & $1 \cdot 10^{-4}$ & - \\
\hline Minimum joint width $\left(t_{\min }\right)$ & - & $1 \cdot 10^{-4}$ & $\mathrm{~m}$ \\
\hline Critical displacement $\left(\delta_{c}\right)$ & - & 0.01 & $\mathrm{~m}$ \\
\hline Yield stress $\left(\sigma_{y}\right)$ & - & $3.5 \cdot 10^{6}$ & $\mathrm{~N} / \mathrm{m}^{2}$ \\
\hline Friction coefficient $\left(\mu_{F}\right)$ & - & 0.4 & - \\
\hline Characteristic length $\left(l_{c}\right)$ & 0.08 & - & $\mathrm{m}$ \\
\hline Propagation length $\left(l_{p}\right)$ & - & 0.05 & $\mathrm{~m}$ \\
\hline Propagation damage $\left(\mathrm{d}_{p}\right)$ & - & 0.4 & - \\
\hline
\end{tabular}

account for the Poisson's effect in the transversal deformation of the soil, a 3D configuration has been chosen for this last test.

As reported in [66], to ensure the efficiency of a hydraulic fracturing process, the spacing between parallel cracks should be of the order of $0.1 \mathrm{~m}$. Here three different spacings have been studied: $s=0.075 \mathrm{~m}, s=0.15 \mathrm{~m}$ and $s=0.3 \mathrm{~m}$, and the crack length in each case has been measured after 125 seconds of simulation. Table 4 summarizes the results along with the obtained crack growth velocity.

The values in Table 4 show that the closer the initial cracks are defined, the faster they propagate, as expected. However, the relation between the spacing and the crack growth velocity seems to be not linear, which also makes sense.

In order to properly appreciate the previous results, the contour lines of the pressure field and the damage variable are represented on two orthogonal planes cutting the cracks (Fig. 13). Looking first at the pressure field in Fig. 13a, c and e, it is quite clear that a closer spacing between cracks makes the pore pressure grow, and consequently the propagation velocity also increases. Also, to understand the interaction between two parallel cracks, it is useful to observe Fig. 13b, $d$ and f. Indeed, the non-local damage model generates a diffusive damage mark around the cracks that extends up to the radius of influence $l_{c}$. In Fig. 13b and d, in which the spacing is smaller than twice the characteristic length of the material, the damage patterns of the cracks overlap each other. In Fig. 13f, with $s>2 l_{c}$, the two damage marks are
Table 4

Parallel fracture propagation test. Crack length and crack growth velocity.

\begin{tabular}{ccc}
\hline Spacing & Crack length at $t=1.5 \mathrm{~s}$ & Crack growth velocity \\
\hline $0.075 \mathrm{~m}$ & $0.4 \mathrm{~m}$ & $0.18 \mathrm{~m} / \mathrm{s}$ \\
$0.15 \mathrm{~m}$ & $0.37 \mathrm{~m}$ & $0.16 \mathrm{~m} / \mathrm{s}$ \\
$0.3 \mathrm{~m}$ & $0.28 \mathrm{~m}$ & $0.1 \mathrm{~m} / \mathrm{s}$ \\
\hline
\end{tabular}

almost independent and hence the velocity of propagation noticeably decreases.

This example shows that the characteristic length of the non-local damage model $l_{c}$ is not only a mathematical parameter used to regularize the strain localization problem, but it actually has a physical meaning.

\section{Conclusions}

A methodology for the $2 \mathrm{D}$ and $3 \mathrm{D}$ analysis of fracture propagation problems in saturated porous media has been presented with the following conclusions:

- The combination of the non-local damage model with the insertion of interface elements can be effectively used in order to propagate fractures in porous media and monitor relevant variables, such as the pressure at the mouth, the crack length and the crack width.

- The integral-type non-local damage model fully regularizes the boundary value problem so that the results are virtually unaffected by changes in the spatial discretization.

- The time step is an important parameter in problems involving material non-linearities and fluid diffusion. A small enough value must be used to ensure accurate results.

- The non-local search of damaged points around the crack tip shows a robust behaviour when predicting the crack path in anisotropic media, including the possibility of branching.

- The FIC-stabilized formulation prevents the blocking of the pressure field under undrained-incompressible conditions and so elements with equal order interpolation for the displacements and the pore pressure can be conveniently used.

- The quasi-zero-thickness interface elements have shown to adequately introduce jumps in the displacement field, and capture the enhanced permeability of the porous medium.

- The proposed technique has been positively tested in a 3D configuration for the analysis of the complex interaction between parallel cracks. As expected, the crack growth velocity is inversely proportional to the spacing between fractures. We note that the memory usage in $3 \mathrm{D}$ problems is remarkable due to the storage of the neighbours for the non-local damage model and thus the computational cost can be intense.

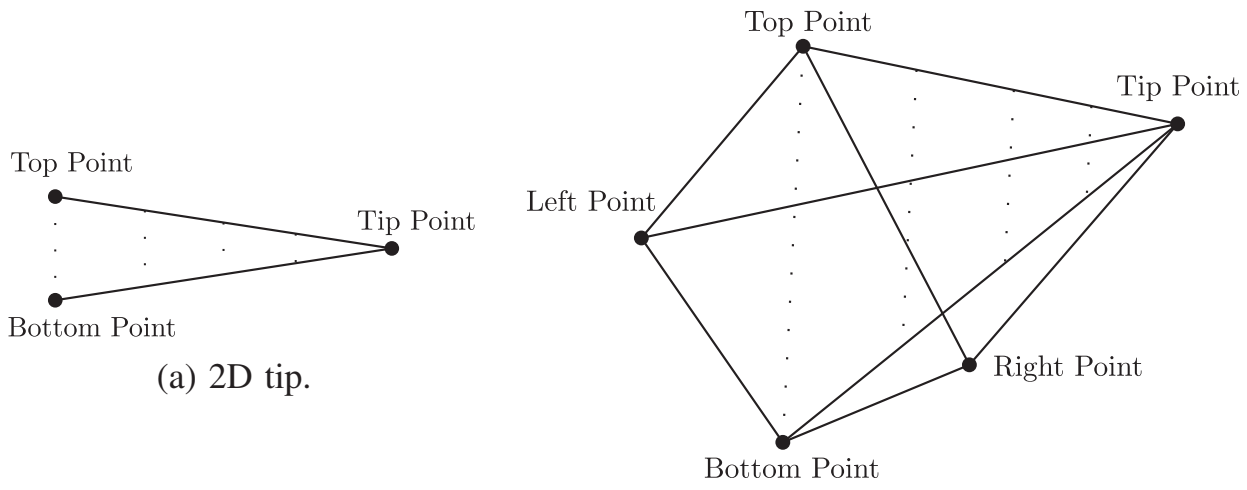

(b) 3D tip.
Fig. 12. Scheme of the implemented crack tips. 


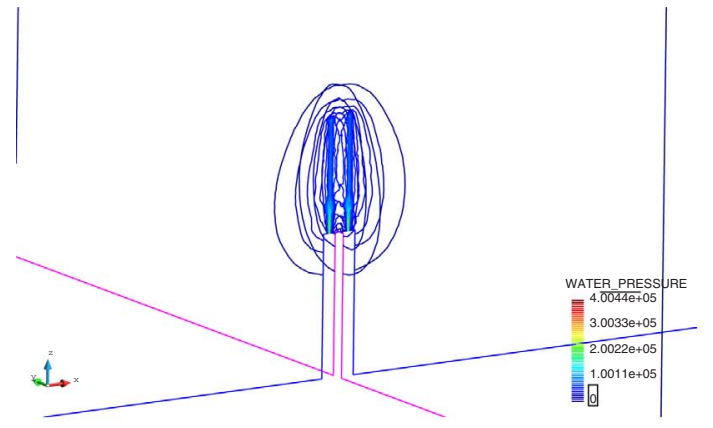

(a) Pressure with $s=0.075 \mathrm{~m}$.

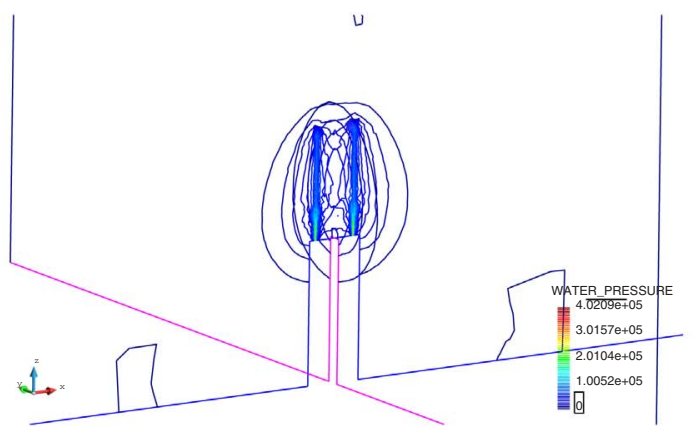

(c) Pressure with $s=0.15 \mathrm{~m}$.

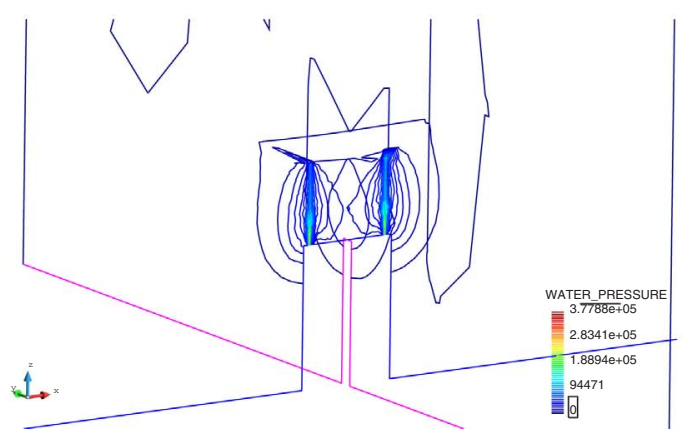

(e) Pressure with $s=0.3 \mathrm{~m}$.

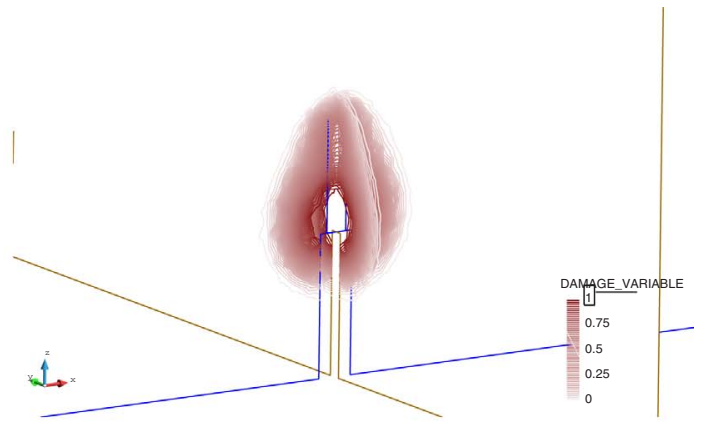

(b) Damage with $s=0.075 \mathrm{~m}$.

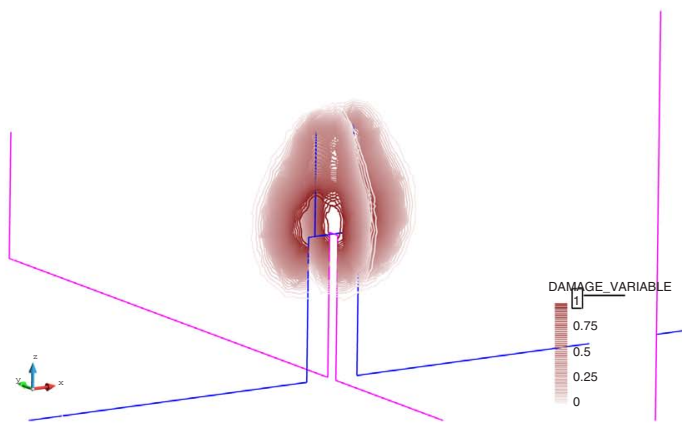

(d) Damage with $s=0.15 \mathrm{~m}$.

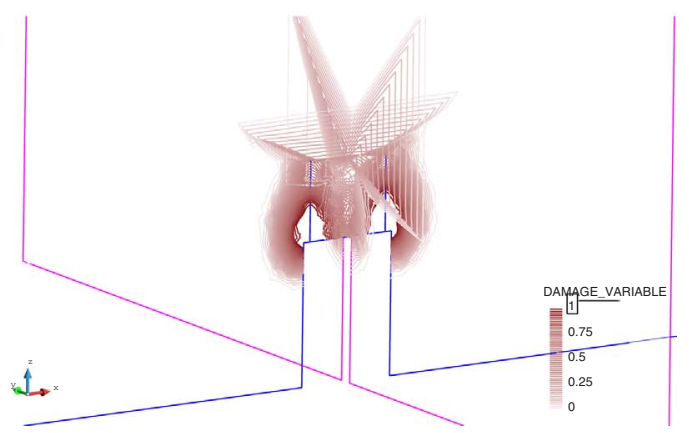

(f) Damage with with $s=0.3 \mathrm{~m}$.

Fig. 13. Parallel fracture propagation test. Pore pressure and damage at $t=1.5 \mathrm{~s}$.

\section{Acknowledgements}

This research was partially funded by the Icebreaker Proof of Concept project of the European Research Council. We are thankful for the financial support to CIMNE via the CERCA Programme of the Generalitat de Catalunya. We also acknowledge the TCAINMAND project as part of this work was performed during a secondment in Tsinghua University.

\section{References}

[1] Adachi J, Siebrits E, Peirce A, Desroches J. Computer simulation of hydraulic fractures. Int J Rock Mech Min Sci 2007;44(5):739-57.

[2] Carrier B, Granet S. Numerical modeling of hydraulic fracture problem in permeable medium using cohesive zone model. Eng Fract Mech 2012;79:312-28.

[3] Zimmermann G, Reinicke A. Hydraulic stimulation of a deep sandstone reservoir to develop an Enhanced Geothermal System: laboratory and field experiments. Geothermics 2010;39(1):70-7.

[4] Jha B, Juanes R. Coupled modeling of multiphase flow and fault poromechanics during geologic $\mathrm{CO}_{2}$ storage. Energy Procedia 2014;63:3313-29.

[5] Casares L, Vincent R, Zalvidea D, Campillo N, Navajas D, Arroyo M, et al. Hydraulic fracture during epithelial stretching. Nat Mater 2015;14(3):343-51.

[6] Lucantonio A, Noselli G, Trepat X, DeSimone A, Arroyo M. Hydraulic fracture and toughening of a brittle layer bonded to a hydrogel. Phys Rev Lett 2015;115(18):188105.

[7] Belytschko T, Black T. Elastic crack growth in finite elements with minimal remeshing. Int J Numer Meth Eng 1999;45(5):601-20.

[8] Réthoré J, De Borst R, Abellan M-A. A two-scale approach for fluid flow in fractured porous media. Int J Numer Meth Eng 2007;71(7):780-800.

[9] Lin Z, Zhuang Z, You X, Wang H, Xu D. Enriched goal-oriented error estimation applied to fracture mechanics problems solved by XFEM. Acta Mech Solida Sin 2012;25(4):393-403.

[10] Zeng Q, Liu Z, Xu D, Wang H, Zhuang Z. Modeling arbitrary crack propagation in coupled shell/solid structures with X-FEM. Int J Numer Meth Eng 2015.

[11] Rashid YR. Ultimate strength analysis of prestressed concrete pressure vessels. Nucl Eng Des 1968;7(4):334-44.

[12] Lubliner J, Oliver J, Oller S, Oñate E. A plastic-damage model for concrete. Int J Solids Struct 1989;25(3):299-326.

[13] Camanho PP, Bessa MA, Catalanotti G, Vogler M, Rolfes R. Modeling the inelastic deformation and fracture of polymer composites - Part II: smeared crack model. Mech Mater 2013;59:36-49.

[14] Heinrich C, Waas AM. Investigation of progressive damage and fracture in laminated composites using the smeared crack approach. CMC-Comput Mater Continua 2013;35:155-81.

[15] Hillerborg A. Numerical methods to simulate softening and fracture of concrete. In: Fracture mechanics of concrete: structural application and numerical calculation; 1985. Springer. p. 141-70.

[16] Cervenka J. Discrete crack modeling in concrete structures. University of Colorado at Boulder; 1994.

[17] Geißler G, Netzker C, Kaliske M. Discrete crack path prediction by an adaptive 
cohesive crack model. Eng Fract Mech 2010;77(18):3541-57.

[18] Etse G, Caggiano A, Vrech S. Multiscale failure analysis of fiber reinforced concrete based on a discrete crack model. Int J Fract 2012;178(1-2):131-46.

[19] Goodman RE, Taylor RL, Brekke TL. A model for the mechanics of jointed rock. J Soil Mech Found Divis 1968.

[20] Andersson J, Dverstorp B. Conditional simulations of fluid flow in three-dimensional networks of discrete fractures. Water Resour Res 1987;23(10):1876-86.

[21] Guiducci C, Pellegrino A, Radu J-P, Collin F, Charlier R. Numerical modeling of hydro-mechanical fracture behavior. In: NUMOG VIII, Balkema; 2002. p. 293-9.

[22] Ng KLA, Small JC. Behavior of joints and interfaces subjected to water pressure. Comput Geotech 1997;20(1):71-93.

[23] Segura JM, Carol I. On zero-thickness interface elements for diffusion problems. Int J Numer Anal Methods Geomech 2004;28(9):947-62.

[24] Barenblatt GI. The formation of equilibrium cracks during brittle fracture. general ideas and hypotheses. Axially-symmetric cracks. J Appl Math Mech 1959;23(3):622-36.

[25] Barenblatt GI. The mathematical theory of equilibrium cracks in brittle fracture. Adv Appl Mech 1962;7(1):55-129.

[26] Dugdale DS. Yielding of steel sheets containing slits. J Mech Phys Solids 1960;8(2):100-4.

[27] Hillerborg A, Modéer M, Petersson P-E. Analysis of crack formation and crack growth in concrete by means of fracture mechanics and finite elements. Cem Concr Res 1976;6(6):773-81.

[28] Camacho GT, Ortiz M. Computational modelling of impact damage in brittle materials. Int J Solids Struct 1996;33(20):2899-938.

[29] Erdogan F, Sih GC. On the crack extension in plates under plane loading and transverse shear. J Fluids Eng 1963;85(4):519-25.

[30] Bouchard PO, Bay F, Chastel Y. Numerical modelling of crack propagation: automatic remeshing and comparison of different criteria. Comput Methods Appl Mech Eng 2003;192(35):3887-908.

[31] Khoei AR, Moslemi H, Ardakany KM, Barani OR, Azadi H. Modeling of cohesive crack growth using an adaptive mesh refinement via the modified-SPR technique. Int J Fract 2009;159(1):21-41.

[32] Sih GC. Strain-energy-density factor applied to mixed mode crack problems. Int J Fract 1974;10(3):305-21.

[33] Hussain MA, Pu SL, Underwood J. Strain energy release rate for a crack under combined mode I and mode II. Fract Anal 1974;560:1.

[34] Van Vroonhoven JCW, De Borst R. Combination of fracture and damage mechanics for numerical failure analysis. Int J Solids Struct 1999;36(8):1169-91.

[35] Moës N, Stolz C, Bernard PE, Chevaugeon N. A level set based model for damage growth: the thick level set approach. Int J Numer Meth Eng 2011;86(3):358-80.

[36] Cazes F, Moës N. Comparison of a phase-field model and of a thick level set model for brittle and quasi-brittle fracture. Int J Numer Meth Eng 2015;103(2):114-43.

[37] de Pouplana I, Oñate E. A FIC-based stabilized mixed finite element method with equal order interpolation for solid-pore fluid interaction problems. Int J Numer Anal Meth Geomech 2017;41(1):110-34.

[38] Biot MA. General theory of three-dimensional consolidation. J Appl Phys 1941;12(2):155-64.

[39] Biot MA, Willis DG. The elastic coefficients of the theory of consolidation. J Appl Mech 1957;24:594-601.

[40] Terzaghi K. Theoretical soil mechanics. New York: Wiley; 1943.

[41] Babuška I. The finite element method with lagrangian multipliers. Numer Math 1973;20(3):179-92.

[42] Brezzi F. On the existence, uniqueness and approximation of saddle-point problems arising from lagrangian multipliers. Revue française d'automatique, informatique, recherche opérationnelle. Analyse numérique 1974;8(2):129-51.

[43] Oñate E. Derivation of stabilized equations for numerical solution of advective- diffusive transport and fluid flow problems. Comput Methods Appl Mech Eng 1998;151(1):233-65.

[44] Oñate E. A stabilized finite element method for incompressible viscous flows using a finite increment calculus formulation. Comput Methods Appl Mech Eng 2000;182(3):355-70.

[45] Oñate E, Taylor RL, Zienkiewicz OC, Rojek J. A residual correction method based on finite calculus. Eng Comput 2003;20(5/6):629-58.

[46] Oñate E, Rojek J, Taylor RL, Zienkiewicz OC. Finite calculus formulation for incompressible solids using linear triangles and tetrahedra. Int J Numer Meth Eng 2004;59(11):1473-500.

[47] Oñate E, Idelsohn SR, Felippa C. Consistent pressure Laplacian stabilization for incompressible continua via higher-order finite calculus. Int J Numer Meth Eng 2011;87(1-5):171-95.

[48] Oñate E, Franci A, Carbonell JM. Lagrangian formulation for finite element analysis of quasi-incompressible fluids with reduced mass losses. Int J Numer Meth Fluids 2014;74(10):699-731.

[49] Zienkiewicz OC, Taylor RL, Zhu JZ. The finite element method 1. 6th ed. Butterworth-Heinemann; 2005.

[50] De Vree JHP, Brekelmans WAM, Van Gils MAJ. Comparison of nonlocal approaches in continuum damage mechanics. Comput Struct 1995;55(4):581-8.

[51] Mazars J. A description of micro-and macroscale damage of concrete structures. Eng Fract Mech 1986;25(5):729-37.

[52] Bazant ZP, Belytschko TB, Chang T-P. Continuum theory for strain-softening. J Eng Mech 1984;110(12):1666-92.

[53] Eringen AC. On nonlocal plasticity. Int J Eng Sci 1981;19(12):1461-74.

[54] Pijaudier-Cabot G, Bazant ZP. Nonlocal damage theory. J Eng Mech 1987;113(10):1512-33.

[55] Rodríguez-Ferran A, Morata I, Huerta A. A new damage model based on non-local displacements. Int J Numer Anal Meth Geomech 2005;29(5):473-93.

[56] Bazant ZP, Jirásek M. Nonlocal integral formulations of plasticity and damage: survey of progress. J Eng Mech 2002;128(11):1119-49.

[57] Song SH, Paulino GH, Buttlar WG. A bilinear cohesive zone model tailored for fracture of asphalt concrete considering viscoelastic bulk material. Eng Fract Mech 2006;73(18):2829-48.

[58] Snow DT. A parallel plate model of fractured permeable media [Ph.D. Thesis]. University of California, Berkeley; 1965.

[59] Witherspoon PA, Wang JSY, Iwai K, Gale JE. Validity of Cubic Law for fluid flow in a deformable rock fracture. Water Resour Res 1980;16(6):1016-24.

[60] Khoei AR, Barani OR, Mofid M. Modeling of dynamic cohesive fracture propagation in porous saturated media. Int J Numer Anal Meth Geomech 2011;35(10):1160-84.

[61] Zhou F, Molinari J-F. Dynamic crack propagation with cohesive elements: a methodology to address mesh dependency. Int J Numer Meth Eng 2004;59(1):1-24.

[62] Barani OR, Khoei AR, Mofid M. Modeling of cohesive crack growth in partially saturated porous media; a study on the permeability of cohesive fracture. Int J Fract 2011;167(1):15-31.

[63] GiD the personal pre and post processor; July 2017. < https://www.gidhome. $\mathrm{com} />$.

[64] de Pouplana I, Oñate E. Combination of a non-local damage model for quasi-brittle materials with a mesh-adaptive finite element technique. Fin Elem Anal Des 2016;112:26-39.

[65] Spence DA, Sharp P. Self-similar solutions for elastohydrodynamic cavity flow. In: Proceedings of the Royal Society of London A: mathematical, physical and engineering sciences, vol. 400. The Royal Society; 1985. p. 289-313.

[66] Chau VT, Bažant ZP, Su Y. Growth model for large branched three-dimensional hydraulic crack system in gas or oil shale. Phil Trans R Soc A 2016;374(2078):20150418. 